arXiv: arXiv:0000.0000

\title{
Bootstrap prediction intervals for Markov processes
}

\author{
Li Pan and Dimitris N. Politis* \\ Li Pan \\ Department of Mathematics \\ University of California-San Diego \\ La Jolla, CA 92093-0112, USA \\ e-mail: lipan@ucsd.edu \\ Dimitris N. Politis \\ Department of Mathematics \\ University of California-San Diego \\ La Jolla, CA 92093-0112, USA \\ e-mail: dpolitis@ucsd.edu
}

\begin{abstract}
Given time series data $X_{1}, \ldots, X_{n}$, the problem of optimal prediction of $X_{n+1}$ has been well-studied. The same is not true, however, as regards the problem of constructing a prediction interval with prespecified coverage probability for $X_{n+1}$, i.e., turning the point predictor into an interval predictor. In the past, prediction intervals have mainly been constructed for time series that obey an autoregressive model that is linear, nonlinear or nonparametric. In the paper at hand, the scope is expanded by assuming only that $\left\{X_{t}\right\}$ is a Markov process of order $p \geq 1$ without insisting that any specific autoregressive equation is satisfied. Several different approaches and methods are considered, namely both Forward and Backward approaches to prediction intervals as combined with three resampling methods: the bootstrap based on estimated transition densities, the Local Bootstrap for Markov processes, and the novel Model-Free bootstrap. In simulations, prediction intervals obtained from different methods are compared in terms of their coverage level and length of interval.
\end{abstract}

Keywords and phrases: Confidence intervals, Local Bootstrap, Model-Free Prediction.

${ }^{*}$ Corresponding author: Dimitris N. Politis, Department of Mathematics, University of California-San Diego, La Jolla, CA 92093-0112, USA; email: dpolitis@ucsd.edu; tel: (858) 534-5861; fax: (858) 534-5273. The website http://www.math.ucsd.edu/ politis/DPsoftware.html contains relevant software for the implementation of methods developed in this paper.

(C) 2015. This manuscript version is made available under the Elsevier user license http://www.elsevier.com/open-access/userlicense/1.0/ 


\section{Introduction}

Prediction is a key objective in time series analysis. The theory of optimal-linear and nonlinearpoint predictors has been well developed. The same is not true, however, as regards the problem of constructing a prediction interval with prespecified coverage probability, i.e., turning the point predictor into an interval predictor. Even in the related problem of regression, the available literature on prediction intervals is not large; see e.g. Geisser (1993), Carroll and Ruppert (1991), Olive (2007), Olive (2015), Patel (1989), Schmoyer (1992), and Stine (1985). Recently, Politis (2013) has re-cast the prediction problem - including prediction intervals - in a Model-Free setting.

An autoregressive (AR) time series model, be it linear, nonlinear, or nonparametric, bears a formal resemblance to the analogous regression model. Indeed, AR models can typically be successfully fitted by the same methods used to estimate a regression, e.g., ordinary Least Square (LS) regression methods for parametric models, and scatterplot smoothing for nonparametric ones. There are several papers for prediction intervals for AR models (typically linear) that represent a broad spectrum of methods; see e.g. Alonso et al. (2002), Box and Jenkins (1976), Breidt et al. (1995), Masarotto (1990), Pascual et al. (2004), Thombs and Schucany (1990), and Wolf and Wunderli (2015).

Recently, Pan and Politis (2015) presented a unified approach towards prediction intervals when a time series $\left\{X_{t}\right\}$ obeys an autoregressive model that is either linear, nonlinear or nonparametric. We expand the scope by assuming only that $\left\{X_{t}\right\}$ is a Markov process of order $p \geq 1$ without insisting that any specific autoregressive equation is satisfied. Recall that Pan and Politis (2015) identified two different general approaches towards building bootstrap prediction intervals with conditional validity, namely the Forward and Backward recursive schemes. We will address both Forward and Backward approaches in the setting of Markovian data; see Section 2 for details.

In terms of the actual resampling mechanism, we will consider the following three options:

1. The bootstrap method based on kernel estimates of the transition density of the Markov processes as proposed by Rajarshi (1990); see Section 3.

2. The Local Bootstrap for Markov processes as proposed by Paparoditis and Politis (1998) and Paparoditis and Politis (2002); see Section 4.

3. The Model-Free Bootstrap for Markov Processes; this is a novel resampling scheme that stems from the Model-Free Prediction Principle of Politis (2013). To elaborate, the key idea is to transform a given complex dataset into one that is i.i.d. (independent, identically distributed); having done that, the prediction problem is greatly simplified, and that includes the construction of prediction intervals. In the case of a Markov Process, this simplification can be accomplished using the Rosenblatt (1952) transformation; see Section 6.

In the case of time series that satisfy an autoregressive equation that is nonlinear and/or nonparametric, Pan and Politis (2015) noted that the Backward approach was not generally feasible. Recall that, under causality, AR models are special cases of Markov processes. Hence, in Section 5 we propose a hybrid approach for nonparametric autoregressions in which the forward step uses the autoregressive equation explicitly while the backward step uses one of the three aforementioned Markov bootstrap procedures.

In the following, Section 2 will describe the setting of the prediction problem under consideration, and the construction of bootstrap prediction intervals. All prediction intervals studied in the paper at hand are asymptotically valid under appropriate conditions. We will assess and compare the finitesample performance of all the methods proposed via Monte Carlo simulations presented in Section 7 . Appendix A is devoted to showing that a Markov process remains Markov after a time-reversal; this is needed to justify the use of all Backward bootstrap approaches. Finally, Appendix B discusses the problem of prediction intervals in $r$-step ahead prediction for $r \geq 1$. 


\section{Prediction and Bootstrap for Markov Processes}

\subsection{Notation and Definitions}

Here, and throughout the rest of the paper, we assume that $X=\left\{X_{t}, t=1,2, \cdots\right\}$ is a real-valued, strictly stationary process that is Markov of order $p$. Letting $Y_{t}=\left(X_{t}, X_{t-1}, \cdots, X_{t-p+1}\right)^{\prime}$, we define

$$
\begin{aligned}
F(y) & =P\left[Y_{p} \leq y\right], \\
F(x, y) & =P\left[X_{p+1} \leq x, Y_{p} \leq y\right], \\
F(x \mid y) & =P\left[X_{p+1} \leq x \mid Y_{p}=y\right],
\end{aligned}
$$

for $x \in \mathbb{R}, y \in \mathbb{R}^{p}$; in the above, we have used the short-hand $\left\{Y_{p} \leq y\right\}$ to denote the event $\{$ the $i$ th coordinate of $Y_{p}$ is less or equal to the $i$ th coordinate of $y$ for all $\left.i=1, \ldots, p\right\}$.

Let $f(y), f(x, y), f(x \mid y)$ be the corresponding densities of the distributions in eq. (2.1). We will assume throughout the paper that these densities are with respect to Lebesgue measure. However, our results in Sections 3 and 4, i.e., bootstrap based on estimated transition densities and Local Bootstrap, could be easily generalized to the case of densities taken with respect to counting measure; i.e., the case of discrete random variables. Remark 6.4 shows a modification that also renders the Model-Free bootstrap of Section 6 valid for discrete data.

Let $X_{1}=x_{1}, X_{2}=x_{2}, \cdots, X_{n}=x_{n}$ denote the observed sample path from the Markov chain $X$, and let $y_{n}=\left(x_{n}, \cdots, x_{n-p+1}\right)^{\prime}$. Denote by $\hat{X}_{n+1}$ the chosen point predictor of $X_{n+1}$ based on the data at hand. Because of the Markov structure, this predictor will be a functional of $\hat{f}_{n}\left(\cdot \mid y_{n}\right)$ which is our data-based estimator of the conditional density $f\left(\cdot \mid y_{n}\right)$. For example, the $L_{2}$-optimal predictor would be given by the mean of $\hat{f}_{n}\left(\cdot \mid y_{n}\right)$; similarly, the $L_{1}$-optimal predictor would be given by the median of $\hat{f}_{n}\left(\cdot \mid y_{n}\right)$. To fix ideas in what follows will will focus on the $L_{2}$-optimal predictor, usually approximated by $\hat{X}_{n+1}=\int x \hat{f}_{n}\left(x \mid y_{n}\right) d x$, with the understanding that other functionals of $\hat{f}_{n}\left(\cdot \mid y_{n}\right)$ can be accommodated equally well.

Remark 2.1. An integral such as $\int x \hat{f}_{n}\left(x \mid y_{n}\right) d x$ can be calculated by numerical integration, e.g. using the adaptive quadrature method. However, the $L_{2}$-optimal predictor can be approximated in several different ways that are asymptotically equivalent. The most straightforward alternative is a kernel smoothed estimator of the autoregression scatterplot, i.e., estimator (5.3) defined in the sequel. Remark 6.2 lists some further alternative options.

Beyond the point predictor $\hat{X}_{n+1}$, we want to construct a prediction interval that will contain $X_{n+1}$ with probability $1-\alpha$ asymptotically; the following definition is helpful.

\section{Definition 2.1. Asymptotic validity of prediction intervals.}

Let $L_{n}, U_{n}$ be functions of the data $X_{1}, \cdots, X_{n}$. The interval $\left[L_{n}, U_{n}\right]$ will be called a $(1-\alpha) 100 \%$ asymptotically valid prediction interval for $X_{n+1}$ given $X_{1}, \cdots, X_{n}$ if

$$
P\left(L_{n} \leq X_{n+1} \leq U_{n}\right) \rightarrow 1-\alpha \text { as } n \rightarrow \infty
$$

for all $\left(X_{1}, \cdots, X_{n}\right)$ in a set that has (unconditional) probability equal to one.

The probability $P$ in (2.2) should be interpreted as conditional probability given $X_{1}, \cdots, X_{n}$ although it is not explicitly denoted; hence, Definition 2.1 indicates conditional validity of the prediction interval $\left[L_{n}, U_{n}\right]$.

Remark 2.2. Asymptotic validity is a fundamental property but it does not tell the whole story; see Pan and Politis (2015) for a full discussion. To elaborate, one could simply let $L_{n}$ and $U_{n}$ be the 
$\alpha / 2$ and $1-\alpha / 2$ of the conditional density estimator $\hat{f}_{n}\left(\cdot \mid y_{n}\right)$ respectively. If $\hat{f}_{n}\left(\cdot \mid y_{n}\right)$ is consistent for $f_{n}\left(\cdot \mid y_{n}\right)$, then this interval would be asymptotically valid; nevertheless, it would be characterized by pronounced under-coverage in finite samples since the nontrivial variability in the estimate $\hat{f}_{n}\left(\cdot \mid y_{n}\right)$ is ignored.

In order to capture the finite-sample variability involved in model estimation some kind of bootstrap algorithm is necessary. Thus, consider a bootstrap pseudo series $X_{1}^{*}, \cdots, X_{n}^{*}$ constructed according to one of the methods mentioned in the Introduction. Let $\hat{f}_{n}^{*}\left(\cdot \mid y_{n}\right)$ be the corresponding estimator of $f\left(\cdot \mid y_{n}\right)$ as obtained from the bootstrap data $X_{1}^{*}, \cdots, X_{n}^{*}$. To achieve conditional validity, we will ensure that the last $p$-values in the bootstrap world coincide with the last $p$-values in the real world, i.e., that $\left(X_{n}^{*}, \cdots, X_{n-p+1}^{*}\right)^{\prime}=y_{n}$. Finally, we construct the predictor $\hat{X}_{n+1}^{*}$ using the same functional, i.e., mean, median, etc., as used in the construction of $\hat{X}_{n+1}$ in the real world but, of course, this time the functional is applied to $\hat{f}_{n}^{*}\left(\cdot \mid y_{n}\right)$. For example, the $L_{2}$-optimal predictor in the bootstrap world will be given by $\hat{X}_{n+1}^{*}=\int x \hat{f}_{n}^{*}\left(x \mid y_{n}\right) d x$.

Bootstrap probabilities and expectations are usually denoted by $P^{*}$ and $E^{*}$, and they are understood to be conditional on the original data $X_{1}=x_{1}, \cdots, X_{n}=x_{n}$. Since Definition 2.1 involves conditional validity, we will understand that $P^{*}$ and $E^{*}$ are also conditional on $X_{n-p+1}^{*}=x_{n-p+1}, \cdots, X_{n}^{*}=x_{n}$ when they are applied to 'future' events in the bootstrap world, i.e., events determined by $\left\{X_{s}^{*}\right.$ for $\left.s>n\right\}$; this is not restrictive since we will ensure that our bootstrap algorithms satisfy this requirement.

Definition 2.2. The predictive root is the error in prediction, i.e., $X_{n+1}-\hat{X}_{n+1}$. Similarly, the bootstrap predictive root is the error in prediction in the bootstrap world, i.e., $X_{n+1}^{*}-\hat{X}_{n+1}^{*}$.

Remark 2.3. Construction of prediction intervals in this paper will be carried out via approximating the quantiles of the predictive root with those of the bootstrap predictive root. To see why, suppose the (conditional) probability $P\left(X_{n+1}-\hat{X}_{n+1} \leq a\right)$ is a continuous function of $a$ in the limit as $n \rightarrow \infty$. If one can show that

$$
\sup _{a}\left|P\left(X_{n+1}-\hat{X}_{n+1} \leq a\right)-P^{*}\left(X_{n+1}^{*}-\hat{X}_{n+1}^{*} \leq a\right)\right| \stackrel{P}{\longrightarrow} 0
$$

then standard results imply that the quantiles of $P^{*}\left(X_{n+1}^{*}-\hat{X}_{n+1}^{*} \leq a\right)$ can be used to consistently estimate the quantiles of $P\left(X_{n+1}-\hat{X}_{n+1} \leq a\right)$, thus leading to asymptotically valid prediction intervals.

Indeed, all prediction intervals that will be studied in this paper are asymptotically valid under appropriate conditions. However, as mentioned earlier, it is difficult to quantify asymptotically the extent to which a prediction interval is able to capture both sources of variation, i.e., the vari-

ance associated with the new observation $X_{n+1}$ and the variability in estimating $\hat{X}_{n+1}$; hence, the prediction intervals in this paper will be compared via finite-sample simulations.

Finally, note that Pan and Politis (2015) also defined prediction intervals based on studentized predictive roots. For concreteness, in this paper we will focus on the simple notion of Definition 2.2 but generalization to studentized predictive roots is straightforward.

\subsection{Forward vs. Backward Bootstrap for Prediction Intervals}

Consider the bootstrap sample $X_{1}^{*}, \cdots, X_{n}^{*}$. As mentioned in Section 2.1, in order to ensure conditional validity it would be helpful if the last $p$-values in the bootstrap world coincided with the last $p$-values in the real world, i.e., that $\left(X_{n}^{*}, \cdots, X_{n-p+1}^{*}\right)^{\prime}=y_{n} \equiv\left(x_{n}, \cdots, x_{n-p+1}\right)^{\prime}$. For the 
application to prediction intervals, note that the bootstrap also allows us to generate $X_{n+1}^{*}$ so that the statistical accuracy of the predictor $\hat{X}_{n+1}$ can be gauged. However, under a usual Monte Carlo simulation, none of the simulated bootstrap series will have their last $p$ values exactly equal to the original vector $y_{n}$ as needed for prediction purposes. Herein lies the problem, since the behavior of the predictor $\hat{X}_{n+1}$ needs to be captured conditionally on the original vector $y_{n}$.

To avoid this difficulty in the set-up of a linear $\operatorname{AR}(p)$ model, Thombs and Schucany (1990) proposed to generate the bootstrap data $X_{1}^{*}, \cdots, X_{n}^{*}$ going backwards from the last $p$ values that are fixed at $\left(X_{n}^{*}, \cdots, X_{n-p+1}^{*}\right)^{\prime}=y_{n}$; this is the backward bootstrap method that was revisited by Breidt et al. (1995) who gave the correct algorithm of finding the backward errors. Note that the generation of $X_{n+1}^{*}$ must still be done in a forward fashion using the fitted AR model conditionally on the value $X_{n}$. Going beyond the linear $\operatorname{AR}(p)$ model, a backward bootstrap for Markov processes was proposed by Paparoditis and Politis (1998) via their notion of Local Bootstrap. We will elaborate on the backward Local Bootstrap and other backward bootstrap methods for Markov processes in the sequel. A key result here it that a Markov process remains Markov after a time-reversal; see our Appendix A.

Nevertheless, the natural way Markov processes evolve is forward in time, i.e., one generates $X_{t}$ given $X_{t-1}, X_{t-2}, \ldots, X_{t-p}$. Thus, it is intuitive to construct bootstrap procedures that run forward in time, i.e., to generate $X_{t}^{*}$ given $X_{t-1}^{*}, X_{t-2}^{*}, \ldots, X_{t-p}^{*}$. Indeed, most (if not all) of the literature on bootstrap confidence intervals for linear AR models uses the natural time order to generate bootstrap series. However, recall that predictive inference is to be conducted conditionally on the last $p$ values given by $y_{n}$ in order to be able to place prediction bounds around the point predictor $\hat{X}_{n+1}$.

In order to maintain the natural time order, i.e., generate bootstrap series forward in time, but also ensure that $X_{n+1}^{*}$ is constructed correctly, i.e., conditionally on the original $y_{n}$, Pan and Politis (2015) introduced the forward bootstrap method for prediction intervals, that comprises of the following two steps. In describing it, we will use the notion of fitting a Markov model by estimating the transition density $f(x \mid y)$ as will be discussed in detail in Section 3; different notions of Markov bootstrap, e.g., the Local Bootstrap, work analogously.

A. Choose a starting vector $\left(X_{1-p}^{*}, X_{2-p}^{*}, \ldots, X_{0}^{*}\right)^{\prime}$ appropriately, e.g., choose it at random as one of the stretches (subseries) of length $p$ found in the original data $X_{1}, \cdots, X_{n}$. Then, use the fitted Markov model, i.e., use the estimated transition density $\hat{f}_{n}(x \mid y)$, in order to generate bootstrap data $X_{t}^{*}$ recursively for $t=1, \ldots, n$. Now re-fit the Markov model using the bootstrap data $X_{1}^{*}, \cdots, X_{n}^{*}$, i.e., obtain $\hat{f}_{n}^{*}(x \mid y)$ as an estimate of the transition density.

B. Re-define the last $p$ values in the bootstrap world, i.e., let $\left(X_{n}^{*}, \cdots, X_{n-p+1}^{*}\right)^{\prime}=y_{n}$, and generate the future bootstrap observation $X_{n+1}^{*}$ by a random draw from density $\hat{f}_{n}^{*}\left(\cdot \mid y_{n}\right)$. Also compute the one-step ahead bootstrap point predictor $\hat{X}_{n+1}^{*}=\int x \hat{f}_{n}^{*}\left(x \mid y_{n}\right) d x$.

Note that the forward bootstrap idea has been previously used for prediction intervals in linear AR models by Masarotto (1990) and Pascual et al. (2004) but with some important differences; for example, Masarotto (1990) omits the important step B above.

Pan and Politis (2015) found that the forward bootstrap is the method that can be immediately generalized to apply for nonlinear and nonparametric autoregressions as well, thus forming a unifying principle for treating all AR models; indeed, for nonlinear and/or nonparametric autoregressions the backward bootstrap seems infeasible. Nevertheless, as will be shown in the next two sections, the backward bootstrap becomes feasible again under the more general setup of Markov process data. In Section 5 we will return briefly to the setup of a nonlinear and/or nonparametric autoregression and propose a hybrid approach in which the forward step uses the autoregressive equation explicitly while the backward step uses one of the three Markov bootstrap procedures mentioned in the Introduction. 


\section{Bootstrap Based on Estimates of Transition Density}

Rajarshi (1990) introduced a bootstrap method that creates pseudo-sample paths of a Markov process based on an estimated transition density; this method can form the basis for a forward bootstrap procedure for prediction intervals. Since the time-reverse of a Markov chain is also a Markov chain - see Appendix A-, it is possible to also define a backward bootstrap based on an estimated backward transition density.

\subsection{Forward Bootstrap Based on Transition Density}

Recall that $x_{1}, x_{2}, \cdots, x_{n}$ is the observed sample path from the Markov chain $X$, and $y_{t}=$ $\left(x_{t}, \cdots, x_{t-p+1}\right)^{\prime}$. In what follows, the phrase "generate $z \sim f(\cdot)$ " will be used as short-hand for "generate $z$ by a random draw from probability density $f(\cdot)$ ".

\section{Algorithm 3.1. Forward Bootstrap}

(1) Choose a probability density $K$ on $\mathbb{R}^{2}$ and positive bandwidths $h_{1}, h_{2}$ to construct the following kernel estimators:

$$
\begin{aligned}
\hat{f}_{n}(x, y) & =\frac{1}{(n-p) h_{1} h_{2}} \sum_{i=p+1}^{n} K\left(\frac{x-x_{i}}{h_{1}}, \frac{\left\|y-y_{i-1}\right\|}{h_{2}}\right) \\
\hat{f}_{n}(y) & =\int \hat{f}_{n}(x, y) d x \\
\hat{f}_{n}(x \mid y) & =\frac{\hat{f}_{n}(x, y)}{\hat{f}_{n}(y)}
\end{aligned}
$$

for all $x \in \mathbb{R}, y \in \mathbb{R}^{p}$, and where $\|\cdot\|$ is a norm in $\mathbb{R}^{p}$.

(2) Calculate the point predictor $\hat{x}_{n+1}=\int x \hat{f}_{n}\left(x \mid y_{n}\right) d x$.

(3) (a) Generate $y_{p}^{*}=\left(x_{p}^{*}, \cdots, x_{1}^{*}\right)$ with probability density function $\hat{f}_{n}(\cdot)$ given by (3.2). Alternatively, let $y_{p}^{*}$ be one of the stretches of $p$ observations that are present as a subset of the original series $x_{1}, \ldots, x_{n}$; there are $n-p+1$ such stretches-choose one of them at random.

(b) Generate $x_{p+1}^{*} \sim \hat{f}_{n}\left(\cdot \mid y_{p}^{*}\right)$ given by (3.3).

(c) Repeat (b) to generate $x_{t+1}^{*} \sim \hat{f}_{n}\left(\cdot \mid y_{t}^{*}\right)$ for $t=p, \cdots, n-1$, where $y_{t}^{*}=\left(x_{t}^{*}, \cdots, x_{t-p+1}^{*}\right)^{\prime}$.

(d) Construct $\hat{f}_{n}^{*}(x \mid y)$ in a similar way as in (3.3) - with the same kernel and bandwidths - but based on the pseudo-data $x_{1}^{*}, x_{2}^{*}, \cdots, x_{n}^{*}$ instead of the original data.

(e) Calculate the bootstrap point predictor $\hat{x}_{n+1}^{*}=\int x \hat{f}_{n}^{*}\left(x \mid y_{n}\right) d x$.

(f) Generate the bootstrap future value $x_{n+1}^{*} \sim \hat{f}_{n}\left(\cdot \mid y_{n}\right)$.

(g) Calculate the bootstrap root replicate as $x_{n+1}^{*}-\hat{x}_{n+1}^{*}$.

(4) Repeat (3) B times; the B bootstrap root replicates are collected in the form of an empirical distribution whose $\alpha$-quantile is denoted $q(\alpha)$.

(5) The $(1-\alpha) 100 \%$ equal-tailed, bootstrap prediction interval for $X_{n+1}$ is given by

$$
\left[\hat{x}_{n+1}+q(\alpha / 2), \hat{x}_{n+1}+q(1-\alpha / 2)\right] .
$$




\subsection{Backward Bootstrap Based on Transition Density}

Letting $Y_{t}=\left(X_{t}, X_{t-1}, \cdots, X_{t-p+1}\right)^{\prime}$ and $x \in \mathbb{R}, y \in \mathbb{R}^{p}$ as before, we can define the backwards transition distribution as $F_{b}(x \mid y)=P\left[X_{0} \leq x \mid Y_{p}=y\right]$ with corresponding density $f_{b}(x \mid y)$. Similarly, we define the backwards joint distribution as $F_{b}(x, y)=P\left[X_{0} \leq x, Y_{p} \leq y\right]$ with corresponding density $f_{b}(x, y)$.

Having observed a sample path $x_{1}, x_{2}, \cdots, x_{n}$ of a Markov chain, Appendix A shows that the time-reversed sample-path $x_{n}, x_{n-1}, \cdots, x_{1}$ can be considered as a sample path of another Markov chain with transition distribution and density given by $F_{b}(x \mid y)=P\left[X_{0} \leq x \mid Y_{p}=y\right]$ and $f_{b}(x \mid y)$ respectively. Note that the densities $f_{b}(x, y)$ and $f_{b}(x \mid y)$ admit kernel estimators as follows:

$$
\begin{aligned}
\hat{f}_{b n}(x, y) & =\frac{1}{(n-p) h_{1} h_{2}} \sum_{i=p+1}^{n} K\left(\frac{x-x_{i-p}}{h_{1}}, \frac{\left\|y-y_{i}\right\|}{h_{2}}\right) \\
\hat{f}_{b n}(x \mid y) & =\frac{\hat{f}_{b n}(x, y)}{\hat{f}_{b n}(y)}
\end{aligned}
$$

The above equation can be used to form an alternative estimator of the unconditional density $f(y)$, namely

$$
\hat{f}_{b n}(y)=\int \hat{f}_{b n}(x, y) d x .
$$

The algorithm for backward bootstrap based on transition density is very similar to that of the corresponding forward bootstrap. The only difference is in Step (3) where we generate the pseudo series $\left(x_{1}^{*}, \cdots, x_{n}^{*}\right)$ in a time-reversed fashion. The backward bootstrap algorithm is described below where the notation $y_{t}^{*}=\left(x_{t}^{*}, \cdots, x_{t-p+1}^{*}\right)^{\prime}$ is again used.

Algorithm 3.2. Backward Bootstrap

(1)-(2) Same as the steps in Algorithm 3.1.

(3) (a) Let $y_{n}^{*}=y_{n}$.

(b) Generate $x_{n-p}^{*} \sim \hat{f}_{b n}\left(\cdot \mid y_{n}^{*}=y_{n}\right)$

(c) Repeat (b) going backwards in time to generate $x_{t}^{*} \sim \hat{f}_{b n}\left(\cdot \mid y_{t+p}^{*}\right)$ for $t=n-p, n-p-1, \cdots, 1$.

(d) Generate bootstrap future value $x_{n+1}^{*} \sim \hat{f}_{n}\left(\cdot \mid y_{n}\right)$. [Note: this is again going forward in time, using the forward transition density exactly as in the Forward Bootstrap Algorithm 3.1.]

(e) Construct $\hat{f}_{n}^{*}(x \mid y)$ in a similar way as in (3.3) - with the same kernel and bandwidths - but based on the pseudo-data $x_{1}^{*}, x_{2}^{*}, \cdots, x_{n}^{*}$ instead of the original data.

(f) Calculate the bootstrap root replicate as $x_{n+1}^{*}-\hat{x}_{n+1}^{*}$.

(4)-(5) Same as the steps in Algorithm 3.1.

\subsection{Asymptotic Properties}

For simplicity, in this section we focus on a Markov sequence $X$ of order one, i.e., $p=1$. The following technical assumptions are needed to ensure asymptotic validity of the prediction intervals of Sections 3.1 and 3.2.

$\left(\alpha_{1}\right) X=\left\{X_{1}, X_{2}, \cdots\right\}$ forms an aperiodic, strictly stationary and geometrically ergodic and $\phi-$ mixing Markov chain on $(\mathbb{R}, \mathcal{B})$ where $\mathcal{B}$ is Borel- $\sigma$ algebra over $\mathbb{R}$. 
$\left(\alpha_{2}\right) F(y), F(x, y)$ and $F(x \mid y)$ defined in eq. (2.1) with $p=1$ are absolutely continuous, and have uniformly continuous and bounded densities $f(y), f(x, y)$ and $f(x \mid y)$ respectively.

$\left(\alpha_{3}\right)$ Assume a compact subset $S \subset \mathbb{R}$ exists such that $f(y) \geq \delta>0$ for each $y \in S$. Also assume $X_{t} \in S$ for all $t \geq 1$.

Remark 2.1 of Rajarshi (1990) provides a discussion on the (non)restrictiveness of assumption $\alpha_{3}$ which should have little effect in practice.

Let $K(x, y)$ be an appropriately chosen probability density on $\mathbb{R}^{2}$; also let $h_{1}=h_{2}=h$ for simplicity. The required conditions on the kernel $K$ and the bandwidth $h$ are specified in assumptions $\left(\beta_{1}-\beta_{3}\right)$.

$\left(\beta_{1}\right) K(x, y)$ is uniformly continuous in $(x, y)$, and $K(x, y) \rightarrow 0$ as $\|(x, y)\| \rightarrow \infty$.

$\left(\beta_{2}\right) K(x, y)$ is of bounded variation on $S \times S$.

$\left(\beta_{3}\right)$ As $n \rightarrow \infty$, we have $h=h(n) \rightarrow 0, n h \rightarrow \infty$ and $\sum_{m=1}^{\infty} m^{k+1} h(m)^{4(k+1)}<\infty$ for some $k \geq 3$.

Under assumptions $\left(\alpha_{1}\right)-\left(\alpha_{3}\right)$ and $\left(\beta_{1}\right)-\left(\beta_{3}\right)$, the following results are proved by Rajarshi (1990).

$$
\begin{aligned}
\sup _{x, y}\left|\hat{f}_{n}(x, y)-f(x, y)\right| \rightarrow 0 & \text { a.s. } \\
\sup _{x}\left|\hat{f}_{n}(x)-f(x)\right| \rightarrow 0 & \text { a.s. } \\
\sup _{x, y}\left|\hat{f}_{n}(x \mid y)-f(x \mid y)\right| \rightarrow 0 & \text { a.s. }
\end{aligned}
$$

Focusing on the forward bootstrap, the above three equations are enough to show that

$$
\sup _{x}\left|P\left(X_{n+1} \leq x\right)-P^{*}\left(X_{n+1}^{*} \leq x\right)\right| \rightarrow 0 \text { a.s. }
$$

To argue in favor of asymptotic validity by appealing to Remark 2.3 , we have to center the distributions appearing in eq. (3.10). Recall that the predictor of future value is $\hat{X}_{n+1}=\int x \hat{f}_{n}\left(x \mid y_{n}\right) d x$, and the bootstrap predictor is $\hat{X}_{n+1}^{*}=\int x \hat{f}_{n}^{*}\left(x \mid y_{n}\right) d x$. Now it is not hard to show that $\hat{X}_{n+1} \rightarrow$ $\int x f\left(x \mid y_{n}\right) d x$ a.s. and $\hat{X}_{n+1}^{*} \rightarrow \int x f\left(x \mid y_{n}\right) d x$ as well; details can be found in Pan (2013). Therefore, it follows that $\hat{X}_{n+1}-\hat{X}_{n+1}^{*} \rightarrow 0$ a.s., and appealing to Remark 2.3 we have the following.

Corollary 3.1. Under assumptions $\left(\alpha_{1}\right)-\left(\alpha_{3}\right)$ and $\left(\beta_{1}\right)-\left(\beta_{3}\right)$, the prediction interval constructed from the forward bootstrap of Algorithm 3.1 is asymptotically valid.

As Appendix A shows, the time-reverse of a Markov process is also a Markov process. Hence, similar arguments leading to Corollary 3.1 can be used to prove the following.

Corollary 3.2. Under assumptions $\left(\alpha_{1}\right)-\left(\alpha_{3}\right)$ and $\left(\beta_{1}\right)-\left(\beta_{3}\right)$, the prediction interval constructed from the backward bootstrap of Algorithm 3.2 is asymptotically valid.

Remark 3.1. [ON BANDwIDTH CHOICE] Bandwidth choice is as difficult as it is important in practice. Rajarshi (1990) used the bandwidth choice $h=0.9 A n^{-1 / 6}$ where $A=\min \left(\hat{\sigma}, \frac{I Q R}{1.34}\right)$, $\hat{\sigma}$ is the estimated standard deviation of the data, and IQR is the interquartile range. However, our simulations indicated that such a bandwidth choice typically gives prediction intervals that exhibit significant over-coverage. Note that the last requirement of assumption $\left(\beta_{3}\right)$ implies that $n h^{4} \rightarrow 0$; this convergence can be very slow provided $k$ is large but in any case the order of $h$ should be at most $O\left(n^{-1 / 4}\right)$. Therefore, the practical bandwidth choice was modified to $h=0.9 A n^{-1 / 4}$. The cross-validation method for bandwidth selection is not recommended here as it results into an $h$ of order $n^{-1 / 5}$. 


\section{The Local Boostrap for Markov processes}

The Local Bootstrap for Markov processes was proposed by Paparoditis and Politis (2002). Although it still assumed that the random variables $X_{1}, X_{2}, \cdots$ are continuous, possessing probability densities, the Local Boostrap generates bootstrap sample paths based on an estimated transition distribution function that is a step function as opposed to generating bootstrap sample paths based on an estimated transition density as in Section 3; in that sense, Rajarshi's (1990) method is to the Local Boostrap what the smoothed bootstrap for i.i.d. data is to Efron's (1979) original bootstrap that resamples from the empirical distribution function.

\subsection{Forward Local Bootstrap}

As before, let $x_{1}, \cdots, x_{n}$ be the observed sample path or a Markov chain of order $p$, and let $Y_{t}=$ $\left(X_{t}, X_{t-1}, \cdots, X_{t-p+1}\right)^{\prime}$ and $y_{t}=\left(x_{t}, x_{t-1}, \cdots, x_{t-p+1}\right)^{\prime}$. Following Paparoditis and Politis (2002), the estimator of the one-step ahead transition distribution function will be given by the weighted empirical distribution

$$
\tilde{F}_{n}(x \mid y)=\frac{\sum_{j=p}^{n-1} 1_{(-\infty, x]}\left(x_{j+1}\right) W_{b}\left(y-y_{j}\right)}{\sum_{m=p}^{n-1} W_{b}\left(y-y_{m}\right)}
$$

where $W_{b}(\cdot)=1 / b W(\cdot / b)$ with $W(\cdot)$ being a bounded, Lipschitz continuous and symmetric probability density kernel in $\mathbb{R}^{p}$, and $b>0$ is a bandwidth parameter tending to zero.

The Local Boostrap generation of pseudo-data will then be based on the estimated conditional distribution $\hat{F}_{n}(x \mid y)$. However, since the latter is a step-function, i.e., it is the distribution of a discrete random variable, in what follows it is easier to work with the probability mass function associated with this discrete random variable.

Algorithm 4.1. Forward Local Bootstrap

(1) Choose a resampling kernel $W$ and bandwidth $b$; here $b$ can be selected by cross validation. Then calculate the predictor $\hat{x}_{n+1}$ as

$$
\frac{\sum_{j=p}^{n-1} W_{b}\left(y_{n}-y_{j}\right) x_{j+1}}{\sum_{m=p}^{n-1} W_{b}\left(y_{n}-y_{m}\right)} .
$$

(2) (a) Set starting value $y_{p}^{*}$ to a subseries consisting of any consecutive $p$ values from $\left\{x_{1}, \cdots, x_{n}\right\}$.

(b) Suppose $x_{1}^{*}, \cdots, x_{t}^{*}$ for $t \geq p$ have already been generated. Let $J$ be a discrete random variable taking its values in the set $\{p, \cdots, n-1\}$ with probability mass function given by

$$
P(J=s)=\frac{W_{b}\left(y_{t}^{*}-y_{s}\right)}{\sum_{m=p}^{n-1} W_{b}\left(y_{t}^{*}-y_{m}\right)} .
$$

Then let $x_{t+1}^{*}=x_{J+1}$ for $t=p$.

(c) Repeat (b) for $t=p+1, p+2, \ldots$ to generate $x_{p+1}^{*}, \cdots, x_{n}^{*}$.

(d) Calculate the bootstrap predictor $\hat{x}_{n+1}^{*}$ as

$$
\frac{\sum_{j=p}^{n-1} W_{b}\left(y_{n}-y_{j}^{*}\right) x_{j+1}^{*}}{\sum_{m=p}^{n-1} W_{b}\left(y_{n}-y_{m}^{*}\right)},
$$

where $y_{t}^{*}=\left(x_{t}^{*}, \cdots, x_{t-p+1}^{*}\right)^{\prime}$. 
(e) Re-define $y_{n}^{*}=y_{n}$, and then generate $x_{n+1}^{*}=x_{J+1}$ as in step (b), where $J$ is a discrete random variable taking its values in the set $\{p, \cdots, n-1\}$ with probability mass function given by

$$
P(J=s)=\frac{W_{b}\left(y_{n}-y_{s}\right)}{\sum_{m=p}^{n-1} W_{b}\left(y_{n}-y_{m}\right)} .
$$

(f) Calculate the bootstrap prediction root replicate as $x_{n+1}^{*}-\hat{x}_{n+1}^{*}$

(3) Repeat step (2) B times; the B bootstrap root replicates are collected in the form of an empirical distribution whose $\alpha$-quantile is denoted $q(\alpha)$.

(4) The $(1-\alpha) 100 \%$ equal-tailed, forward Local Bootstrap prediction interval for $X_{n+1}$ is given by

$$
\left[\hat{x}_{n+1}+q(\alpha / 2), \hat{x}_{n+1}+q(1-\alpha / 2)\right] .
$$

\subsection{Backward Local Bootstrap}

As shown in Appendix A, the time-reverse of a Markov process is also a Markov process. So in this section we will employ the Backward Local Bootstrap that was introduced by Paparoditis and Politis (1998) for the purpose of constructing prediction intervals; their example was a first order autoregressive process with conditionally heteroscedastic errors, i.e., the model $X_{t}=\phi X_{t-1}+$ $\epsilon_{t} \sqrt{\alpha_{0}+\alpha_{1} X_{t-1}^{2}}$ where $\left\{\epsilon_{t}\right\}$ are i.i.d with mean 0 and variance 1 .

We will now generalize this idea to the $\operatorname{Markov}(p)$ case; in what follows, the Backward Local Bootstrap employs an estimate of the backward conditional distribution given by

$$
\tilde{F}_{b n}(x \mid y)=\frac{\sum_{j=1}^{n-p} 1_{(-\infty, x]}\left(x_{j}\right) W_{b^{\prime}}\left(y-y_{j+p}\right)}{\sum_{m=1}^{n-p} W_{b^{\prime}}\left(y-y_{m+p}\right)}
$$

where $b^{\prime}$ is the backward bandwidth which can be different from the forward bandwidth $b$.

Algorithm 4.2. Backward Local Bootstrap

(1) Same as the Forward Local Bootstrap in Algorithm 4.1.

(2) (a) Set starting value $y_{n}^{*}=y_{n}$.

(b) Suppose $y_{t+p}^{*}$ has already been generated where $1 \leq t \leq n-p$. Let $J$ be a discrete random variable taking its values in the set $\{1,2, \cdots, n-p\}$ with probability mass function given by

$$
P(J=s)=\frac{W_{b^{\prime}}\left(y_{t+p}^{*}-y_{s+p}\right)}{\sum_{m=1}^{n-p} W_{b^{\prime}}\left(y_{t+p}^{*}-y_{m+p}\right)} .
$$

Then let $x_{t}^{*}=x_{J}$.

(c) Repeat (b) to generate $x_{n-p}^{*}, \cdots, x_{2}^{*}, x_{1}^{*}$ backwards in time, i.e., first generate $x_{n-p}^{*}$, then generate $x_{n-p-1}^{*}$, etc.

(d) Let $J$ be a discrete random variable taking its values in the set $\{p, p+1, \cdots, n-1\}$ with probability mass function given by

$$
P(J=s)=\frac{W_{b}\left(y_{n}-y_{s}\right)}{\sum_{m=p}^{n-1} W_{b}\left(y_{n}-y_{m}\right)} .
$$

Then let $x_{n+1}^{*}=x_{J+1}$. [Note: this is again going forward in time exactly as in the Forward Local Bootstrap Algorithm 4.1.] 
(e) Calculate the bootstrap predictor $\hat{x}_{n+1}^{*}$ by

$$
\frac{\sum_{j=p}^{n-1} W_{b}\left(y_{n}-y_{j}^{*}\right) x_{j+1}^{*}}{\sum_{m=p}^{n-1} W_{b}\left(y_{n}-y_{m}^{*}\right)}
$$

(f) Calculate the bootstrap prediction root replicate as $x_{n+1}^{*}-\hat{x}_{n+1}^{*}$.

(3)-(4) Same as the steps of the Forward Local Bootstrap Algorithm 4.1.

\subsection{Asymptotic Properties}

Paparoditis and Politis (2002) under their assumptions (A1)-(A3) and (B1)-(B2) proved that

$$
\sup _{x, y}\left|\tilde{F}_{n}(x \mid y)-F(x \mid y)\right| \rightarrow 0 \text { a.s. }
$$

where the transition distribution estimator $\tilde{F}(x \mid y)$ was defined in (4.1); this is sufficient to show eq. (3.10) for the Forward Local Bootstrap. The argument regarding $\hat{X}_{n+1}-\hat{X}_{n+1}^{*} \rightarrow 0$ is similar as in Section 3.3, and hence asymptotic validity follows.

Corollary 4.1. Under assumptions (A1)-(A3) and (B1)-(B2) of Paparoditis and Politis (2002), the prediction interval constructed from the Forward Local Bootstrap of Algorithm 4.1 is asymptotically valid.

Due to Appendix A, it is easy to see that the same consistency can be shown for the backwards transition distribution estimator $\tilde{F}_{b n}(y \mid x)$ defined in (4.2), i.e., that

$$
\sup _{x, y}\left|\tilde{F}_{b n}(x \mid y)-F_{b}(x \mid y)\right| \rightarrow 0 \text { a.s. }
$$

from which asymptotic validity of the Backward Local Bootstrap follows.

Corollary 4.2. Under assumptions (A1)-(A3) and (B1)-(B2) of Paparoditis and Politis (2002), the prediction interval constructed from the Backward Local Bootstrap of Algorithm 4.2 is asymptotically valid.

\section{Hybrid Backward Markov Bootstrap for Nonparametric Autoregression}

In this section only, we will consider the special case where our $\operatorname{Markov}(p)$ process is generated via a nonparametric autoregression model, i.e., one of the two models below:

- AR with homoscedastic errors:

$$
X_{t}=m\left(X_{t-1}, \ldots, X_{t-p}\right)+\epsilon_{t} \text { with } \epsilon_{t} \sim \text { i.i.d. }\left(0, \sigma^{2}\right)
$$

- AR with heteroscedastic errors:

$$
X_{t}=m\left(X_{t-1}, \ldots, X_{t-p}\right)+\sigma\left(X_{t-1}, \ldots, X_{t-p}\right) \epsilon_{t} \text { with } \epsilon_{t} \sim \text { i.i.d. }(0,1) .
$$

As before, we assume that $\left\{X_{t}\right\}$ is strictly stationary; we further need to assume causality, i.e., that $\epsilon_{t}$ is independent of $\left\{X_{t-1}, X_{t-2}, \ldots\right\}$ for all $t$. As usual, the recursions (5.1) and (5.2) are meant to 
run forward in time, i.e., $X_{p+1}$ is generated given an initial assignment for $X_{1}, \ldots, X_{p}$; then, $X_{p+2}$ is generated given its own $p$-past, etc.

Recently, Pan and Politis (2015) presented a unified approach for prediction intervals based on forward, model-based resampling under one of the above two models. It was noted that a backward model-based bootstrap is not feasible except in the special case where the conditional expectation function $m\left(X_{t-1}, \ldots, X_{t-p}\right)$ is affine in its arguments, i.e., a linear AR model; see e.g. Breidt et al. (1995) and Thombs and Schucany (1990).

Using the ideas presented in Sections 3 and 4, we can now propose a hybrid Backward Markov Bootstrap for Nonparametric Autoregression in which forward resampling is done using the model, i.e., eq. (5.1) or (5.2), whereas the backward resampling is performed using the Markov property only.

\subsection{Hybrid Backward Markov Bootstrap Algorithms}

Consider Markov Processes generated from either homoscedastic model (5.1) or heteroscedastic model (5.2). Given a sample $\left\{x_{1}, x_{2}, \cdots, x_{n}\right\}$, the algorithms of hybrid backward Markov bootstrap based on transition density for nonparametric model with i.i.d errors and heteroscedastic errors are described in Algorithm 5.1 and 5.2 respectively; the corresponding algorithms based on Local Bootstrap are described in Algorithm 5.3 and 5.4.

Algorithm 5.1. Hybrid backward Backward Markov bootstrap based on transition density for nonparametric model with homoscedastic errors

(1) Select a bandwidth $h$ and construct the kernel estimates $\hat{m}\left(y_{i}\right)$ for $i=p, \cdots, n-1$, where

$$
\hat{m}\left(y_{i}\right)=\frac{\sum_{t=p}^{n-1} K\left(\frac{\left\|y_{i}-y_{t}\right\|}{h}\right) x_{t+1}}{\sum_{t=p}^{n-1} K\left(\frac{\left\|y_{i}-y_{t}\right\|}{h}\right)} ;
$$

as before, $y_{t}=\left(x_{t}, x_{t-1}, \cdots, x_{t-p+1}\right)^{\prime}$, and $\|\cdot\|$ is a norm in $\mathbb{R}^{p}$.

(2) Compute the residuals: $\hat{\epsilon}_{i}=x_{i}-\hat{m}\left(y_{i-1}\right)$, for $i=p+1, \cdots, n$.

(3) Center the residuals: $\hat{r}_{i}=\hat{\epsilon}_{i}-(n-p)^{-1} \sum_{t=p+1}^{n} \hat{\epsilon_{t}}$, for $i=p+1, \cdots$, n; let the empirical distribution of $\hat{r}_{t}$ denoted by $\hat{F}_{\epsilon}$.

(a) Construct the backward transition density estimate $\hat{f}_{b n}$ as eq. (3.6).

(b) Let $y_{n}^{*}=y_{n}$.

(c) Generate $x_{n-p}^{*} \sim \hat{f}_{b n}\left(\cdot \mid y_{n}^{*}=y_{n}\right)$. Repeat it to generate $x_{t}^{*} \sim \hat{f}_{b n}\left(\cdot \mid y_{t+p}^{*}\right)$ for $t=n-p, \cdots, 1$ backwards in time, i.e., first for $t=n-p$, then for $t=n-p-1$, etc.

(d) Compute the future bootstrap observation $X_{n+1}^{*}$ by the AR formula

$$
X_{n+1}^{*}=\hat{m}\left(y_{n}^{*}\right)+\epsilon_{n+1}^{*}=\hat{m}\left(y_{n}\right)+\epsilon_{n+1}^{*},
$$

where $\epsilon_{n+1}^{*}$ is generated from $\hat{F}_{\epsilon}$. Then re-estimate $m(\cdot)$ based on the pseudo data, i.e.,

$$
\hat{m}^{*}(y)=\frac{\sum_{i=p}^{n-1} K\left(\frac{\left\|y-y_{i}^{*}\right\|}{h}\right) x_{i+1}^{*}}{\sum_{i=p}^{n-1} K\left(\frac{\left\|y-y_{i}^{*}\right\|}{h}\right)},
$$

and let $\hat{X}_{n+1}^{*}=\hat{m}^{*}\left(y_{n}^{*}\right)=\hat{m}^{*}\left(y_{n}\right)$ 
(e) Calculate the bootstrap root replicate as $X_{n+1}^{*}-\hat{X}_{n+1}^{*}$.

(4) Steps (a)-(e) in the above are repeated $B$ times, and the B bootstrap root replicates are collected in the form of an empirical distribution whose $\alpha$-quantile is denoted $q(\alpha)$.

(5) Then, a $(1-\alpha) 100 \%$ equal-tailed predictive interval for $X_{n+1}$ is given by

$$
\left[\hat{m}\left(x_{n}\right)+q(\alpha / 2), \hat{m}\left(x_{n}\right)+q(1-\alpha / 2)\right]
$$

Algorithm 5.2. Hybrid backward Markov bootstrap based on transition density for nonparametric model with heteroscedastic errors

(1) Select the bandwidth $h$ and construct the estimates $\left\{\hat{m}\left(y_{i}\right), \hat{\sigma}\left(y_{i}\right)\right\}$ for $i=p, \cdots, n-1$, where

$$
\begin{gathered}
\hat{m}\left(y_{i}\right)=\frac{\sum_{t=p}^{n-1} K\left(\frac{\left\|y_{i}-y_{t}\right\|}{h}\right) x_{t+1}}{\sum_{t=p}^{n-1} K\left(\frac{\left\|y_{i}-y_{t}\right\|}{h}\right)}, \\
\hat{\sigma}^{2}\left(y_{i}\right)=\frac{\sum_{t=1}^{n-1} K\left(\frac{\left\|y_{i}-y_{t}\right\|}{h}\right)\left(x_{t+1}-\hat{m}\left(y_{t}\right)\right)^{2}}{\sum_{t=1}^{n-1} K\left(\frac{\left\|y_{i}-y_{t}\right\|}{h}\right)} .
\end{gathered}
$$

(2) Compute the residuals:

$$
\hat{\epsilon_{i}}=\frac{x_{i}-\hat{m}\left(y_{i-1}\right)}{\hat{\sigma}\left(y_{i-1}\right)},
$$

for $i=p+1, \cdots, n$.

(3) This step is similar as step (3) in Algorithm 5.1; the only difference is in step (d) when the future bootstrap observation $X_{n+1}^{*}$ is computed as follows:

$$
X_{n+1}^{*}=\hat{m}_{g}\left(y_{n}^{*}\right)+\hat{\sigma}_{g}\left(y_{n}^{*}\right) \epsilon_{n+1}^{*}=\hat{m}_{g}\left(y_{n}\right)+\hat{\sigma}_{g}\left(y_{n}\right) \epsilon_{n+1}^{*}
$$

Here $\hat{m}_{g}$ and $\hat{\sigma}_{g}$ are over-smoothed estimates of $m$ and $g$ computed in the same way as $\hat{m}$ and $\hat{\sigma}$ but using bandwidth $g$ that is bigger than $h$; see Pan and Politis (2015) for a discussion.

(4)-(5) Same as those in Algorithm 5.1.

Algorithm 5.3. Hybrid backward local bootstrap for nonparametric model with homoscedastic errors

The algorithm is identical to Algorithm 5.1 with the exception of steps 3 (a) to (c) that have to be changed as follows.

(3) (a)' Select a resampling bandwidth $b^{\prime}$ and kernel $W$.

$(b)^{\prime}$ Let $y_{n}^{*}=y_{n}$ Suppose $y_{t+p}^{*}$ have already been generated for $1 \leq t \leq n-p$. Let $J$ be a discrete random variable taking its values in the set $\{1,2, \cdots, n-p\}$ with probability mass function given by

$$
P(J=s)=\frac{W_{b^{\prime}}\left(y_{t+p}^{*}-y_{s+p}\right)}{\sum_{m=1}^{n-p} W_{b^{\prime}}\left(y_{t+p}^{*}-y_{m+p}\right)},
$$

Then $x_{t}^{*}=x_{J}$.

$(c)^{\prime}$ Repeat (b) to generate $x_{n-p}^{*}, \cdots, x_{2}^{*}, x_{1}^{*}$ backwards in time.

Algorithm 5.4. Hybrid backward local bootstrap for nonparametric model with heteroscedastic errors 
(1)-(2) Same as the corresponding steps in Algorithm 5.2

(3) (a)-(c) Same as steps $(a)^{\prime}-(c)^{\prime}$ in Algorithm 5.3

(d)-(e) Same as the corresponding steps in Algorithm 5.2

(4)-(5) Same as the corresponding steps in Algorithm 5.2

Remark 5.1. The hybrid algorithms 5.1 -5.4 use a model-based resampling based on fitted residuals. As discussed by Pan and Politis (2015), usage of predictive residuals may be preferable. According to the two models (5.1) or (5.2), the predictive residuals are respectively defined as $\hat{\epsilon}_{t}^{(t)}=x_{t}-\hat{m}_{t}^{(t)}\left(y_{t-1}\right)$ or $\hat{\epsilon}_{t}^{(t)}=\frac{x_{t}-\hat{m}_{t}^{(t)}\left(y_{t-1}\right)}{\hat{\sigma}_{t}^{(t)}\left(y_{t-1}\right)}$ where $\hat{m}^{(t)}$ and $\hat{\sigma}^{(t)}$ are smoothing estimators calculated from the original dataset having the $t$-th point deleted. Finally, to define hybrid backward bootstrap intervals based on predictive residuals we just need to replace the fitted residuals $\left\{\hat{\epsilon}_{i}\right\}$ in step (2) of Algorithms 5.1-5.4 by the predictive residuals $\left\{\hat{\epsilon}_{t}^{(t)}\right\}$.

\section{Bootstrap Prediction Intervals for Markov Processes Based on the Model-Free Prediction Principle}

We now return to the setup of data from a general Markov process that does not necessarily satisfy a model equation such as (5.1) or (5.2). In what follows, we will introduce the Model-Free Bootstrap for Markov Processes; this is a novel approach that stems from the Model-Free Prediction Principle of Politis (2013). The key idea is to transform a given complex dataset into one that is i.i.d., and therefore easier to handle; having done that, the prediction problem is greatly simplified, and this includes the construction of prediction intervals. In the case of a Markov Process, this simplification can be practically accomplished using the Rosenblatt (1952) transformation.

Instead of generating one-step ahead pseudo data by some estimated conditional distribution, e.g. the transition density given in (3.3) or the transition distribution function given in (4.1), the Model-Free Bootstrap resamples the transformed i.i.d. data, and then transforms them back to obtain the desired one-step ahead prediction. Note that the bootstrap based on kernel estimates of the transition density of Section 3, and the Local Bootstrap of Section 4 can also be considered model-free methods as they apply in the absence of a model equation such as (5.1) or (5.2). The term Model-Free Bootstrap specifically refers to the transformation approach stemming from the Model-Free Prediction Principle of Politis (2013).

\subsection{Theoretical Transformation}

Let $X=\left\{X_{1}, X_{2}, \cdots\right\}$ be a stationary time series from a Markov process of order $p$, and $Y_{t-1}=$ $\left(X_{t-1}, \cdots, X_{t-p}\right)^{\prime}$. Given $Y_{t-1}=y \in \mathbf{R}^{p}$, we denote the conditional distribution of $X_{t}$ as

$$
D_{y}(x)=P\left(X_{t} \leq x \mid Y_{t-1}=y\right) .
$$

This is the same distribution discussed in eq. (2.1); changing the notation will help us differentiate between the different methods. For some positive integer $i \leq p$, we also define the distributions with partial conditioning as follows

$$
D_{y, i}(x)=P\left(X_{t} \leq x \mid Y_{t-1}^{(i)}=y\right)
$$

where $Y_{t-1}^{(i)}=\left(X_{t-1}, \cdots, X_{t-i}\right)^{\prime}$ and $y \in \mathbf{R}^{i}$. In this notation, we can denote the unconditional distribution as $D_{y, 0}(x)=P\left(X_{t} \leq x\right)$ which does not depend on $y$. Throughout this section, we assume that, for any $y$ and $i$, the function $D_{y, i}(\cdot)$ is continuous and invertible over its support. 
A transformation from our $\operatorname{Markov}(p)$ dataset $X_{1}, \cdots, X_{n}$ to an i.i.d. dataset $\eta_{1}, \cdots, \eta_{n}$ can now be constructed as follows. Let

$$
\begin{gathered}
\eta_{1}=D_{y, 0}\left(X_{1}\right) ; \eta_{2}=D_{Y_{1}^{(1)}, 1}\left(X_{2}\right) ; \eta_{3}=D_{Y_{2}^{(2)}, 2}\left(X_{3}\right) ; \cdots ; \eta_{p}=D_{Y_{p-1}^{(p-1)}, p-1}\left(X_{p}\right) \\
\quad \text { and } \eta_{t}=D_{Y_{t-1}}\left(X_{t}\right) \text { for } t=p+1, p+2, \cdots, n .
\end{gathered}
$$

Note that the transformation from the vector $\left(X_{1}, \cdots, X_{m}\right)^{\prime}$ to the vector $\left(\eta_{1}, \cdots, \eta_{m}\right)^{\prime}$ is one-to-one and invertible for any natural number $m$ by construction. Hence, the event $\left\{X_{1}=x_{1}, \cdots, X_{t}=x_{m}\right\}$ is identical to the event $\left\{\eta_{1}=\zeta_{1}, \cdots, \eta_{t}=\zeta_{m}\right\}$ when the construction of $\zeta_{t}$ follows (6.3) and (6.4), i.e.,

$$
\begin{gathered}
\zeta_{1}=D_{y, 0}\left(x_{1}\right) ; \zeta_{2}=D_{y_{1}^{(1)}, 1}\left(x_{2}\right) ; \zeta_{3}=D_{y_{2}^{(2)}, 2}\left(x_{3}\right) ; \cdots ; \zeta_{p}=D_{Y_{p-1}^{(p-1)}, p-1}\left(x_{p}\right) \\
\text { and } \zeta_{t}=D_{y_{t-1}}\left(x_{t}\right) \text { for } t=p+1, p+2, \cdots, n
\end{gathered}
$$

where $y_{t-1}=\left(x_{t-1}, \cdots, x_{t-p}\right)^{\prime}$ and $y_{t-1}^{(i)}=\left(x_{t-1}, \cdots, x_{t-i}\right)^{\prime}$.

It is not difficult to see that the random variables $\eta_{1}, \cdots, \eta_{n}$ are i.i.d. Uniform( 0,1$)$; in fact, this is an application of the Rosenblatt (1952) transformation in the case of $\operatorname{Markov}(p)$ sequences. For example, the fact that $\eta_{1}$ is Uniform $(0,1)$ is simply due to the probability integral transform. Now for $t>p$, we have $P\left(\eta_{t} \leq z \mid \eta_{t-1}=\zeta_{t-1}, \ldots, \eta_{1}=\zeta_{1}\right)=P\left(\eta_{t} \leq z \mid X_{t-1}=x_{t-1}, \ldots, X_{1}=x_{1}\right)=$ $P\left(\eta_{t} \leq z \mid Y_{t-1}=y_{t-1}, X_{t-p-1}=x_{t-p-1}, \ldots, X_{1}=x_{1}\right)$ by the discussion preceding eq. (6.5). Letting $y$ be a short-hand for $y_{t-1}$, we have:

$$
\begin{aligned}
P\left(\eta_{t} \leq z \mid \eta_{t-1}=\zeta_{t-1}, \ldots, \eta_{1}=\zeta_{1}\right) & =P\left(D_{y}\left(X_{t}\right) \leq z \mid Y_{t-1}=y, X_{t-p-1}=x_{t-p-1}, \ldots, X_{1}=x_{1}\right) \\
& =P\left(X_{t} \leq D_{y}^{-1}(z) \mid Y_{t-1}=y\right) \quad \text { (by the Markov property) } \\
& =D_{y}\left(D_{y}^{-1}(z)\right) \\
& =z \quad \text { (which is uniform and does not depend on } y) .
\end{aligned}
$$

Hence, $P\left(\eta_{t} \leq z \mid \eta_{t-1}=\zeta_{t-1}, \ldots, \eta_{1}=\zeta_{1}\right)=z$, i.e., for $t>p, \eta_{t}$ is a random variable that is independent of the its own past and has a Uniform $(0,1)$ distribution. The same is true for $\eta_{t}$ with $1<t<p$; the argument is similar to the above but using the $D_{y, t}(\cdot)$ distribution instead of $D_{y}(\cdot)$. All in all, it should be clear that the random variables $\eta_{1}, \cdots, \eta_{n}$ are i.i.d. Uniform $(0,1)$.

\subsection{Estimating the Transformation from Data}

To estimate the theoretical transformation from data, we would need to estimate the distributions $D_{y, i}(\cdot)$ for $i=0,1, \ldots, p-1$ and $D_{y}(\cdot)$. Note, however, that $D_{y, i}(\cdot)$ for $i<p$ can-in principle - be computed from $D_{y}(\cdot)$ since the latter uniquely specifies the whole distribution of the stationary Markov process. Hence, it should be sufficient to just estimate $D_{y}(\cdot)$ from our data. Another way of seeing this is to note that the $p$ variables in eq. (6.3) can be considered as 'edge effects' or 'initial conditions'; the crucial part of the transformation is given by eq. (6.4), i.e., the one based on $D_{y}(\cdot)$.

Given observations $x_{1}, \cdots, x_{n}$, we can estimate $D_{y}(x)$ by local averaging methods such as the kernel estimator

$$
\hat{D}_{y}(x)=\frac{\sum_{i=p+1}^{n} 1_{\left\{x_{i} \leq x\right\}} K\left(\frac{\left\|y-y_{i-1}\right\|}{h}\right)}{\sum_{k=p+1}^{n} K\left(\frac{\left\|y-y_{k-1}\right\|}{h}\right)} .
$$


Note that $\hat{D}_{y}$ in (6.7) is a step function; we can use linear interpolation on this step function to produce an estimate $\tilde{D}_{y}$ that is piecewise linear and strictly increasing (and therefore invertible); see Politis (2010, Section 4.1) for details on this linear interpolation. Consequently, we define

$$
u_{t}=\tilde{D}_{y_{t-1}}\left(x_{t}\right) \text {, for } t=p+1, \cdots, n \text {. }
$$

Estimator $\hat{D}_{y}$ is consistent for $D_{y}$ under regularity conditions; see eq. (4.3) and the associated discussion. Furthermore, the consistency of $\tilde{D}_{y}$ follows from its close proximity to $\hat{D}_{y}$; see the related discussion in Politis (2010).

It then follows that $u_{t} \approx \eta_{t}$ where $\eta_{t}$ was defined in Section 6.1 , and thus $\left\{u_{t}\right.$ for $\left.t=p+1, \cdots, n\right\}$ are approximately i.i.d. Uniform $(0,1)$. Hence, the goal of transforming our data $x_{1}, \cdots, x_{n}$ to a sequence of (approximately) i.i.d. random variables $u_{t}$ has been achieved; note that the 'initial conditions' $u_{1}, \ldots, u_{p}$ were not explicitly generated in the above as they are not needed in the Model-free bootstrap algorithms.

\subsection{Basic Algorithm for Model-free Prediction Interval}

Given observations $x_{1}, \cdots, x_{n}$, the basic model-free (MF) algorithm for constructing the predictive interval can be described as follows. As before, $y_{t-1}=\left(y_{t-1}, \cdots, y_{t-p}\right)^{\prime}$ and $y_{t-1}^{*}=\left(x_{t-1}^{*}, \cdots, x_{t-p}^{*}\right)^{\prime}$.

Algorithm 6.1. Model-Free (MF) Method

(1) Use eq. (6.8) to obtain the transformed data $u_{p+1}, \cdots, u_{n}$.

(2) Calculate $\hat{x}_{n+1}$, the predictor of $x_{n+1}$, by

$$
\hat{x}_{n+1}=\frac{1}{n-p} \sum_{t=p+1}^{n} \tilde{D}_{y_{n}}^{-1}\left(u_{t}\right) .
$$

(3) (a) Resample randomly(with replacement) the transformed data $u_{p+1}, \cdots, u_{n}$ to create the pseudo data $u_{-M}^{*}, u_{-M+1}^{*}, \cdots, u_{0}^{*}, u_{1}^{*}, \cdots, u_{n-1}^{*}, u_{n}^{*}$ and $u_{n+1}^{*}$ for some large positive integer $M$.

(b) Draw $x_{-M}^{*}, \cdots, x_{-M+p-1}^{*}$ randomly from any consecutive $p$ values of the dataset $\left(x_{1}, \cdots, x_{n}\right) ;$ let $y_{-M+p-1}^{*}=\left(x_{-M+p-1}^{*}, \cdots, x_{-M}^{*}\right)^{\prime}$.

(c) Generate $x_{t}^{*}=\tilde{D}_{y_{t-1}^{*}}^{-1}\left(u_{t}^{*}\right)$ for $t=-M+p, \cdots, n$.

(d) Calculate the bootstrap future value $x_{n+1}^{*}=\tilde{D}_{y_{n}}^{-1}\left(u_{n+1}^{*}\right)$.

(e) Calculate the bootstrap predictor $\hat{x}_{n+1}^{*}=\frac{1}{n-p} \sum_{t=p+1}^{n} \tilde{D}_{y_{n}}^{*-1}\left(u_{t}^{*}\right)$ where $\tilde{D}_{y}^{*}$ is a linearly interpolated version of the step function given by

$$
\hat{D}_{y}^{*}(x)=\frac{\sum_{i=p+1}^{n} 1_{\left\{x_{i}^{*} \leq x\right\}} K\left(\frac{\left\|y-y_{i-1}^{*}\right\|}{h}\right)}{\sum_{k=p+1}^{n} K\left(\frac{\left\|y-y_{k-1}^{*}\right\|}{h}\right)} .
$$

(f) Calculate the bootstrap root $x_{n+1}^{*}-\hat{x}_{n+1}^{*}$.

(4) Repeat step (3) B times; the B bootstrap root replicates are collected in the form of an empirical distribution whose $\alpha$-quantile is denoted $q(\alpha)$.

(5) The $(1-\alpha) 100 \%$ equal-tailed predictive interval for $X_{n+1}$ is given by

$$
\left[\hat{x}_{n+1}+q(\alpha / 2), \hat{x}_{n+1}+q(1-\alpha / 2)\right] .
$$


Some remarks are in order.

Remark 6.1. Algorithm 6.1 is in effect a Forward bootstrap algorithm for prediction intervals according to the discussion of Section 2. Constructing a backward bootstrap analog of Algorithm 6.1 is straightforward based on the Markov property of the reversed Markov process shown in Appendix A. One would then need a reverse construction of the theoretical transformation of Section 6.1. To elaborate on the latter, we would instead let $\eta_{t}=G_{Y_{t+p}}\left(X_{t}\right)$ for $t=n-p, n-p-1, \ldots, 1$ where $G_{y}(x)=P\left(X_{t} \leq x \mid Y_{t+p}=y\right)$ is the backwards analog of $D_{y}(x)$; the $\eta_{t}$ for $t=n, \ldots, n-p+1$ can be generated using the backwards analogs of $D_{y, i}(x)$. The details are straightforward and are omitted especially since the finite-sample performance of the two approaches is practically indistinguishable.

Remark 6.2. As mentioned in Section 2, there exist different approximations to the conditional expectation which serves as the $L_{2}$-optimal predictor. The usual one is the kernel smoothed estimator (5.3) but eq. (6.9) gives an alternative approximation; we have used it in Algorithm 6.1 because it follows directly from the Model-Free Prediction Principle of Politis (2013). However, the two approximations are asymptotically equivalent, and thus can be used interchangeably. To see why, note that

$\frac{1}{n-p} \sum_{t=p+1}^{n} \tilde{D}_{y_{n}}^{-1}\left(u_{t}\right) \approx \int_{0}^{1} \tilde{D}_{y_{n}}^{-1}(u) d u \approx \int x \hat{f}_{n}\left(x \mid y_{n}\right) d x \approx \hat{m}\left(y_{n}\right)=\int_{0}^{1} \hat{D}_{y_{n}}^{-1}(u) d u \approx \frac{1}{n-p} \sum_{t=p+1}^{n} \hat{D}_{y_{n}}^{-1}\left(u_{t}\right)$

where $\hat{D}_{y}^{-1}(\cdot)$ indicates the quantile inverse of the step-function $\hat{D}_{y}(\cdot)$.

Remark 6.3. Recall that $\hat{D}_{y}(x)$ is a local average estimator, i.e., averaging the indicator $1_{\left\{x_{i} \leq x\right\}}$ over data vectors $Y_{t}$ that are close to $y$. If $y$ is outside the range of the data vectors $Y_{t}$, then obviously estimator $\hat{D}_{y}(x)$ can not be constructed, and the same is true for $\tilde{D}_{y}(x)$. Similarly, if $y$ is at the edges of the range of $Y_{t}$, e.g., within $h$ of being outside the range, then $\hat{D}_{y}(x)$ and $\tilde{D}_{y}(x)$ will not be very accurate. Step 1 of Algorithm 6.1 can then be modified to drop the $u_{i}$ s that are obtained from an $x_{i}$ whose $y_{i-1}$ is within $h$ of the boundary; see Politis (2013) for a related discussion.

Remark 6.4. [Discrete-VAlued Markov processes] Since the transformed data $u_{p+1}, \ldots, u_{n}$ are approximately i.i.d. Uniform(0,1), the resampling in step 3(a) of Algorithm 6.1 could alternatively be done using the Uniform distribution, i.e., generate $u_{-M}^{*}, u_{-M+1}^{*}, \cdots, u_{0}^{*}, u_{1}^{*}, \cdots, u_{n-1}^{*}, u_{n}^{*}$ and $u_{n+1}^{*}$ as i.i.d. Uniform(0,1). Algorithm 6.1 still works fine with this choice but can not obviously be extended to include the use of "predictive residuals" as Section 6.4 proposes; see also the discussion in the Rejoinder of Politis (2013). Interestingly, generating $u_{i}^{*}$ as i.i.d. Uniform(0,1), and replacing all occurences of $\tilde{D}_{y}^{-1}(\cdot)$ by the quantile inverse $\hat{D}_{y}^{-1}(\cdot)$ in Algorithm 6.1 , makes the algorithm valid for the situation where the time series $X_{t}$ is discrete-valued, i.e., when the true $D_{y}(x)$ is indeed a step function in $x$.

\subsection{Better Model-free Prediction Intervals: the Predictive Model-free Method}

In Pan and Politis (2015), model-based predictive residuals were used instead of the fitted residuals to improve the performance of the predictive interval. From eq. (6.7), we see that the conditional distribution of interest is $D_{y_{n}}(x)=P\left(X_{t} \leq x \mid Y_{t-1}=y_{n}\right)$ which is estimated by

$$
\hat{D}_{y_{n}}(x)=\frac{\sum_{i=p+1}^{n} 1_{\left\{x_{i} \leq x\right\}} K\left(\frac{\left\|y_{n}-y_{i-1}\right\|}{h}\right)}{\sum_{k=p+1}^{n} K\left(\frac{\left\|y_{n}-y_{i-1}\right\|}{h}\right)} .
$$


Since $x_{n+1}$ is not observed, the above estimated conditional distribution for $x_{n+1}$ treats the pair $\left(y_{n}, x_{n+1}\right)$ as an "out-of-sample" pair. To mimic this situation in the model-free set-up, we can use the trick of Pan and Politis (2015), i.e., to calculate an estimate of $D_{y_{n}}(x)$ based on a dataset that excludes the pair $\left(y_{t-1}, x_{t}\right)$ for $t=p+1, \cdots, n$. In other words, define the 'delete-one' estimator

$$
\hat{D}_{y_{t-1}}^{(t)}\left(x_{t}\right)=\frac{\sum_{i=p+1, i \neq t}^{n} 1_{\left\{x_{i} \leq x_{t}\right\}} K\left(\frac{\left\|y_{t-1}-y_{i-1}\right\|}{h}\right)}{\sum_{k=p+1, k \neq t} K\left(\frac{\left\|y_{t-1}-y_{k-1}\right\|}{h}\right)} \text {, for } t=p+1, \cdots, n .
$$

Linear interpolation on $\hat{D}_{y}^{(t)}(x)$ gives $\tilde{D}_{y}^{(t)}(x)$, and we can then define

$$
u_{t}^{(t)}=\tilde{D}_{y_{t-1}}^{(t)}\left(x_{t}\right)
$$

here, the $u_{t}^{(t)}$ serve as the analogs of the predictive residuals studied in Pan and Politis (2015) in a nonparametric regression setup.

Algorithm 6.2. Predictive Model-Free (PMF) Method

The algorithm is identical to Algorithm 6.1 after substituting $u_{p+1}^{(p+1)}, \cdots, u_{n}^{(n)}$ in place of $u_{p+1}, \cdots, u_{n}$.

Remark 6.5. If $y_{t-1}$ is far from other $y_{i}$ s (for $i=p+1, \cdots, n$ and $i \neq t$ ), then the denominator of $\hat{D}_{y_{t-1}}^{(t)}\left(x_{t}\right)$ can be zero which leads to an undefined value of $u_{t}^{(t)}$. We omit all these undefined $u_{t}^{(t)} \mathrm{s}$ in the practical application of the above algorithm.

\subsection{Smoothed Model-Free Method}

In Section 6.2, we estimated the transition distribution $D_{y}(x)=P\left(X_{t} \leq x \mid Y_{t-1}=y\right)$ by $\hat{D}_{y}(x)$ as defined in (6.7). Noting that $D_{y}(x)$ is, by assumption, continuous in $x$ while $\hat{D}_{y}(x)$ is not, the linearly interpolated, strictly increasing estimator $\tilde{D}_{y}(x)$ was used instead. However, $\tilde{D}_{y}(x)$ is piecewice linear, and therefore not smooth in the argument $x$. In this section, we employ an alternative estimator of the conditional transition density that is smooth in $x$.

Note that the function $1_{\left\{x_{i} \leq x\right\}}$ is the cumulative distribution of a point mass distribution. To smooth this point mass distribution, we substitute $1_{\left\{x_{i} \leq x\right\}}$ in eq. (6.7) with $\Lambda\left(\frac{x-x_{i}}{h_{0}}\right)$ where $\Lambda(\cdot)$ is an absolutely continuous, strictly increasing cumulative distribution function, and $h_{0}$ is a positive bandwidth parameter. The new estimator $\bar{D}_{y}(x)$ is defined by

$$
\bar{D}_{y}(x)=\frac{\sum_{i=p+1}^{n} \Lambda\left(\frac{x-x_{i}}{h_{0}}\right) K\left(\frac{\left\|y-y_{i-1}\right\|}{h}\right)}{\sum_{k=p+1}^{n} K\left(\frac{\left\|y-y_{k-1}\right\|}{h}\right)},
$$

and the transformed data $\left\{v_{t}\right.$ for $\left.t=p+1, \cdots, n\right\}$ can be calculated by

$$
v_{t}=\bar{D}_{y_{t-1}}\left(x_{t}\right) \text {. }
$$

Substituting $\bar{D}_{y}(x)$ for $\tilde{D}_{y}(x)$ and $\left\{v_{t}\right\}$ for $\left\{u_{t}\right\}$ in Algorithm 6.1, we have the smoothed model free method as follows:

Algorithm 6.3. Smoothed Model-Free Method (SMF)

(1) Use eq. (6.12) to obtain the transformed data $v_{p+1}, \cdots, v_{n}$. 
(2) Calculate $\hat{x}_{n+1}$, the predictor of $x_{n+1}$, by

$$
\hat{x}_{n+1}=\frac{1}{n-p} \sum_{t=p+1}^{n} \bar{D}_{x_{n}}^{-1}\left(v_{t}\right)
$$

(3) (a) Resample randomly (with replacement) the transformed data $v_{p+1}, \cdots, v_{n}$ to create the pseudo data $v_{-M}^{*}, v_{-M+1}^{*}, \cdots, v_{0}^{*}, v_{1}^{*}, \cdots, v_{n-1}^{*}, v_{n}^{*}$ and $v_{n+1}^{*}$ for some large positive integer $M$.

(b) Draw $x_{-M}^{*}, \cdots, x_{-M+p-1}^{*}$ from any consecutive $p$ values of the dataset $\left(x_{1}, \cdots, x_{n}\right)$; let $y_{-M+p-1}^{*}=\left(x_{-M+p-1}^{*}, \ldots, x_{-M}^{*}\right)$.

(c) Generate $x_{t}^{*}=\bar{D}_{y_{t-1}^{*}}^{-1}\left(v_{t}^{*}\right)$ for $t=-M+p, \cdots, n$.

(d) Calculate the bootstrap future value $x_{n+1}^{*}=\bar{D}_{y_{n}}^{-1}\left(v_{n+1}^{*}\right)$.

(e) Calculate $\hat{x}_{n+1}^{*}=\frac{1}{n-p} \sum_{t=p+1}^{n} \bar{D}_{y_{n}}^{*-1}\left(v_{t}^{*}\right)$ where

$$
\bar{D}_{y}^{*}(x)=\frac{\sum_{i=p+1}^{n} \Lambda\left(\frac{x-x_{i}^{*}}{h_{0}}\right) K\left(\frac{\left\|y-y_{i-1}^{*}\right\|}{h}\right)}{\sum_{k=p+1}^{n} K\left(\frac{\left\|y-y_{k-1}^{*}\right\|}{h}\right)} .
$$

(f) Calculate the bootstrap root $x_{n+1}^{*}-\hat{x}_{n+1}^{*}$.

(4) Repeat step (3) B times; the B bootstrap root replicates are collected in the form of an empirical distribution whose $\alpha$-quantile is denoted $q(\alpha)$.

(5) The $(1-\alpha) 100 \%$ equal-tailed predictive interval for $X_{n+1}$ is given by

$$
\left[\hat{x}_{n+1}+q(\alpha / 2), \hat{x}_{n+1}+q(1-\alpha / 2)\right] \text {. }
$$

Remark 6.6. As in Remark 6.3, Step 1 (a) of Algorithm 6.3 can be modified to drop the $v_{i}$ s that are obtained from an $x_{i}$ s whose $y_{i-1}$ is within $h$ of the boundary.

Remark 6.7. [ON BAndwidth ChOICE] As suggested by Li and Racine (2007) Chapter 6.2, the optimal smoothing of $\bar{D}_{y}(x)$ with respect to Mean Squared Error (MSE) requires that $h_{0}=$ $O_{p}\left(n^{-2 / 5}\right)$ and $h=O_{p}\left(n^{-1 / 5}\right)$; hence, in the algorithm's implementation, we chose $h$ through cross validation, and then let $h_{0}=h^{2}$.

As in Section 6.4, we can also use the delete- $x_{t}$ estimator

$$
\bar{D}_{y}^{(t)}\left(x_{t}\right)=\frac{\sum_{i=p+1, i \neq t}^{n} \Lambda\left(\frac{x_{t}-x_{i}}{h_{0}}\right) K\left(\frac{\left\|y_{t-1}-y_{i-1}\right\|}{h}\right)}{\sum_{k=p+1, k \neq t} K\left(\frac{\left\|y_{t-1}-y_{k-1}\right\|}{h}\right)} \text { for } t=p+1, \cdots, n
$$

in order to construct the transformed data:

$$
v_{t}^{(t)}=\bar{D}_{y_{t-1}}^{(t)}\left(x_{t}\right) \text { for } t=p+1, \cdots, n .
$$

This leads to the Predictive Smoothed Model-Free Algorithm.

Algorithm 6.4. Predictive Smoothed Model-Free (PSMF) Method

The algorithm is identical to Algorithm 6.3 after substituting $v_{p+1}^{(p+1)}, \cdots, v_{n}^{(n)}$ in place of $v_{p+1}, \cdots, v_{n}$. 


\subsection{Why is Smoothing Advisable?}

To fix ideas, let us take $p=1$ and $x, y \in \mathbb{R}$; then, the unsmoothed (step-function) estimator of $D_{y}(x)$ is given by

$$
\hat{D}_{y}(x)=\frac{\sum_{i=1}^{n-1} 1_{\left\{V_{i} \leq x\right\}} K\left(\frac{y-U_{i}}{h}\right)}{\sum_{k=1}^{n-1} K\left(\frac{y-U_{k}}{h}\right)}
$$

whereas the smoothed (differentiable) one is

$$
\bar{D}_{y}(x)=\frac{\sum_{i=1}^{n-1} \Lambda\left(\frac{V_{i}-x}{h_{0}}\right) K\left(\frac{y-U_{i}}{h}\right)}{\sum_{k=1}^{n-1} K\left(\frac{y-U_{k}}{h}\right)} ;
$$

in the above, we have used the notation $\left(V_{i}, U_{i}\right)=\left(x_{i+1}, x_{i}\right)$ for $i=1,2, \ldots$. Note that this is tantamount to estimating the conditional distribution in the nonparametric (auto)regression of the variable $V_{i}$ on the variable $U_{i}$ since both $\hat{D}_{y}(x)$ and $\bar{D}_{y}(x)$ are estimates of $P\left(V_{i} \leq x \mid U_{i}=y\right)$.

Carrying out a nonparametric regression of a response variable $V_{i}$ on a regressor variable $U_{i}$ is formally the same whether the scatterplot pairs $\left\{\left(V_{i}, U_{i}\right)\right.$ for $\left.i=1,2, \ldots\right\}$ are i.i.d. or dependent (as in our Markov case). In fact, the large-sample distributions of estimators in nonparametric autoregression often turn out to be identical to the corresponding ones of nonparametric regression with i.i.d. scatterplot pairs; see e.g. Kreiss and Neumann (1998).

In the case of i.i.d. scatterplot pairs $\left\{\left(V_{i}, U_{i}\right)\right.$ for $\left.i=1,2, \ldots\right\}$, estimators (6.13) and (6.14) have been extensively studied. In particular, when $D_{y}(x)$ is very smooth, e.g. twice continuously differentiable in $x$, Li and Racine (2007) Chapter 6.1 showed that

$$
0<M S E\left[\hat{D}_{y}(x)\right]-M S E\left[\bar{D}_{y}(x)\right]=O\left(\frac{h_{0}}{n h}\right)=o\left(\frac{1}{n h}\right) ;
$$

recall that $M S E\left[\hat{D}_{y}(x)\right]=O\left(\frac{1}{n h}\right)$ and $M S E\left[\bar{D}_{y}(x)\right]=O\left(\frac{1}{n h}\right)$ as well.

Consequently, it follows that $\bar{D}_{y}(x)$ is more accurate than either $\hat{D}_{y}(x)$ or $\tilde{D}_{y}(x)$, i.e., that smoothing may indeed be advisable. It is conjectured that a similar phenomenon takes place when the scatterplot pairs $\left\{\left(V_{i}, U_{i}\right)\right.$ for $\left.i=1,2, \ldots\right\}$ are dependent as in the case where $\left(V_{i}, U_{i}\right)=\left(x_{i+1}, x_{i}\right)$ with $x_{1}, x_{2}, \ldots$ being a realization of a Markov process. This conjecture is empirically confirmed in the next section where it will be apparent that the smoothed model free (SMF) method has better performance in simulations.

\section{Finite-sample Simulations}

In this final section, we use Monte Carlo simulations to assess the performance of the bootstrap methods proposed in this paper through average coverage level(CVR) and length(LEN).

For each type of bootstrap method, we generate 500 datasets each with size $n$. And for each data set, we use the bootstrap method to create $B$ bootstrap sample paths and $B$ one-step ahead future values $\left(Y_{n+1, j}, j=1,2, \cdots, B\right)$. We construct replicates of the bootstrap prediction interval $\left(L_{i}, U_{i}\right)$, $i=1,2, \cdots, 500$, and estimate its coverage level and length by

$$
C V R=\frac{1}{500} \sum_{i=1}^{500} C V R_{i} \text { and } L E N=\frac{1}{500} \sum_{i=1}^{500} L E N_{i}
$$


where

$$
C V R_{i}=\frac{1}{B} \sum_{j=1}^{B} 1_{\left[L_{i}, U_{i}\right]}\left(Y_{n+1, j}\right) \text { and } L E N_{i}=U_{i}-L_{i} .
$$

Note that for the $i$-th data set of size $n$ (where $i=1,2, \cdots, 500$ ), we have the prediction interval $\left(L_{i}, U_{i}\right)$ of $X_{n+1}$ given $X_{n}=x_{n i}$, where $x_{n i}$ is the last observation from the $i$-th data set. The values of these $x_{n i} \mathrm{~s}$ are different for each $i$; therefore, the above $C V R$ is an estimate of unconditional, i.e., average, coverage level.

To evaluate the performance of the different bootstrap methods, the following models were chosen in order to generate Markov process data (of order $p=1$ ).

- Model 1: $X_{t+1}=\sin \left(X_{t}\right)+\epsilon_{t+1}$

- Model 2: $X_{t+1}=0.8 \log \left(3 X_{t}^{2}+1\right)+\epsilon_{t+1}$

- Model 3: $X_{t+1}=-0.5 \exp \left(-50 X_{t}^{2}\right) X_{t}+\epsilon_{t+1}$

- Model 4: $X_{t+1}=\sin \left(X_{t}\right)+\sqrt{0.5+0.25 X_{t}^{2}} \epsilon_{t+1}$

- Model 5: $X_{t+1}=0.75 X_{t}+0.15 X_{t} \epsilon_{t+1}+\epsilon_{t+1}$

In the above, the errors $\left\{\epsilon_{t}\right\}$ are chosen either from i.i.d. $N(0,1)$ distribution or i.i.d. Laplace distribution rescaled to variance 1 . We choose sample sizes $n=50,100$ and 200, and constructed $95 \%$ and $90 \%$ prediction intervals. In the bootstrap procedures, we choose $B=250$.

For the bootstrap approach based on the transition density, we chose $K(x, y)=k_{1}(x) k_{1}(y)$ where $k_{1}(x)$ is the standard normal density. As suggested in Remark 3.1, we chose $h$ to be $0.9 A n^{-1 / 4}$ where $A=\min \left(\hat{\sigma}, \frac{I Q R}{1.34}\right), \hat{\sigma}$ is the estimated standard deviation of the data, and IQR is the interquartile range.

The kernel $W$ in the local bootstrap method is the normal kernel, and the forward and backward bandwidth $b$ and $b^{\prime}$ are chosen by cross validation method.

In the hybrid backward bootstrap procedure for nonparametric autoregression, the estimation bandwidth $h$ for nonparametric bootstrap and the resampling bandwidth $b$ for local bootstrap are all selected by cross validation based on corresponding regression kernel estimators. As in Section 3, the resampling bandwidth $b$ for the backward bootstrap based on the transition density is given by $0.9 A n^{-1 / 4}$. The over-smoothing bandwidth we used is $g=2 h$; cf. Pan and Politis (2015).

For the model-free methods, we used the standard normal cumulative distribution function restricted on $[-2,2]$ as $\Lambda(\cdot)$. We chose bandwidth $h$ for kernel $K(\cdot)$ through cross validation and chose the smoothing bandwidth $h_{0}$ to be $h_{0}=h^{2}$ as mentioned in Remark 6.7.

Tables 1-10 summarize all the simulation results. The first two lines of each table are the simulation results using a model-based bootstrap procedure introduced in Pan and Politis (2015) where the practitioner fits a nonparametric AR model to the data, and resamples the residuals; entries nonpara-f and nonpara-p denote resampling the fitted vs. predictive residuals respectively. All our data generating mechanisms happen to follow nonparametric AR models; thus, the first two lines of each table should serve as benchmarks for comparison since model-based methods should have an advantage when the model happens to be true.

Line 3 and 4 of each table are the simulation results using bootstrap based on transition density discussed in Section 3; the forward and backward methods are denoted trans-forward and trans-backward respectively. Lines 5,6 are the results using the local bootstrap discussed in Section 4; the forward and backward methods are denoted lbs-forward and lbs-backward respectively.

Line 7 to 10 are the simulation results using the hybrid backward Markov bootstrap for nonparametric autoregression discussed in Section 5. Notation hybrid-trans-f and hybrid-trans-p denote that the backward generating mechanism uses an estimator of transition density while the generation of $X_{n+1}^{*}$ is done via model-based resampling the fitted vs. predictive residuals respectively. 
Similarly, notation hybrid-lbs-f and hybrid-lbs-p denote that the backward generating mechanism is done by local bootstrap while the generation of $X_{n+1}^{*}$ is done via model-based resampling the fitted vs. predictive residuals respectively.

The last four lines of each table summarize the simulation results using all the model free methods discussed in Section 6. We used both the basic model-free (MF) method of Algorithm 6.1 as well as the Predictive MF of Algorithm 6.2; the notation is MF-fitted and MF-predictive respectively. Similarly for the Smoothed Model-Free Methods of Section 6.5; the notation is SMF-fitted and SMF-predictive respectively.

\begin{tabular}{|c|c|c|c|c|c|c|}
\hline Normal innovations & \multicolumn{3}{|c|}{ nominal coverage $95 \%$} & \multicolumn{3}{|c|}{ nominal coverage $90 \%$} \\
\hline$n=50$ & CVR & LEN & st.err & CVR & LEN & st.err \\
\hline nonpara-f & 0.913 & 3.824 & 0.519 & 0.855 & 3.223 & 0.424 \\
\hline nonpara-p & 0.941 & 4.207 & 0.536 & 0.893 & 3.547 & 0.425 \\
\hline trans-forward & 0.931 & 4.126 & 0.760 & 0.886 & 3.544 & 0.667 \\
\hline trans-backward & 0.931 & 4.130 & 0.757 & 0.887 & 3.555 & 0.677 \\
\hline lbs-forward & 0.910 & 3.885 & 0.778 & 0.862 & 3.337 & 0.685 \\
\hline lbs-backward & 0.911 & 3.920 & 0.795 & 0.863 & 3.355 & 0.676 \\
\hline Hybrid-trans-f & 0.908 & 3.822 & 0.522 & 0.852 & 3.230 & 0.432 \\
\hline Hybrid-trans-p & 0.935 & 4.181 & 0.583 & 0.889 & 3.553 & 0.470 \\
\hline Hybrid-lbs-f & 0.914 & 3.782 & 0.525 & 0.860 & 3.199 & 0.433 \\
\hline Hybrid-lbs-p & 0.938 & 4.136 & 0.592 & 0.892 & 3.496 & 0.463 \\
\hline MF-fitted & 0.893 & 3.647 & 0.740 & 0.844 & 3.150 & 0.645 \\
\hline MF-predictive & 0.926 & 4.108 & 0.854 & 0.880 & 3.482 & 0.721 \\
\hline SMF-fitted & 0.892 & 3.627 & 0.717 & 0.843 & 3.131 & 0.619 \\
\hline SMF-predictive & 0.939 & 4.293 & 0.828 & 0.893 & 3.614 & 0.709 \\
\hline$n=100$ & CVR & LEN & st.err & CVR & LEN & st.err \\
\hline nonpara-f & 0.927 & 3.860 & 0.393 & 0.873 & 3.255 & 0.310 \\
\hline nonpara-p & 0.943 & 4.099 & 0.402 & 0.894 & 3.456 & 0.317 \\
\hline trans-forward & 0.942 & 4.137 & 0.627 & 0.901 & 3.535 & 0.519 \\
\hline trans-backward & 0.942 & 4.143 & 0.621 & 0.900 & 3.531 & 0.519 \\
\hline lbs-forward & 0.930 & 3.980 & 0.625 & 0.886 & 3.409 & 0.511 \\
\hline lbs-backward & 0.932 & 4.001 & 0.605 & 0.886 & 3.411 & 0.508 \\
\hline Hybrid-trans-f & 0.921 & 3.822 & 0.412 & 0.868 & 3.241 & 0.335 \\
\hline Hybrid-trans-p & 0.936 & 4.045 & 0.430 & 0.889 & 3.441 & 0.341 \\
\hline Hybrid-lbs-f & 0.923 & 3.815 & 0.430 & 0.869 & 3.226 & 0.343 \\
\hline Hybrid-lbs-p & 0.937 & 4.018 & 0.433 & 0.890 & 3.414 & 0.338 \\
\hline MF-fitted & 0.917 & 3.771 & 0.602 & 0.869 & 3.238 & 0.497 \\
\hline MF-predictive & 0.941 & 4.152 & 0.680 & 0.897 & 3.511 & 0.550 \\
\hline SMF-fitted & 0.916 & 3.731 & 0.551 & 0.869 & 3.221 & 0.489 \\
\hline SMF-predictive & 0.946 & 4.231 & 0.647 & 0.902 & 3.471 & 0.530 \\
\hline$n=200$ & CVR & LEN & st.err & CVR & LEN & st.err \\
\hline nonpara-f & 0.938 & 3.868 & 0.272 & 0.886 & 3.263 & 0.219 \\
\hline nonpara-p & 0.948 & 4.012 & 0.283 & 0.899 & 3.385 & 0.231 \\
\hline trans-forward & 0.944 & 4.061 & 0.501 & 0.902 & 3.472 & 0.415 \\
\hline trans-backward & 0.944 & 4.058 & 0.507 & 0.902 & 3.470 & 0.424 \\
\hline lbs-forward & 0.937 & 3.968 & 0.530 & 0.891 & 3.369 & 0.439 \\
\hline lbs-backward & 0.937 & 3.979 & 0.551 & 0.893 & 3.383 & 0.448 \\
\hline Hybrid-trans-f & 0.932 & 3.838 & 0.359 & 0.880 & 3.238 & 0.290 \\
\hline Hybrid-trans-p & 0.942 & 3.977 & 0.360 & 0.893 & 3.358 & 0.281 \\
\hline Hybrid-lbs-f & 0.932 & 3.798 & 0.336 & 0.882 & 3.228 & 0.272 \\
\hline Hybrid-lbs-p & 0.942 & 3.958 & 0.338 & 0.895 & 3.356 & 0.265 \\
\hline MF-fitted & 0.924 & 3.750 & 0.481 & 0.878 & 3.222 & 0.403 \\
\hline MF-predictive & 0.942 & 4.065 & 0.558 & 0.894 & 3.395 & 0.455 \\
\hline SMF-fitted & 0.924 & 3.731 & 0.464 & 0.877 & 3.208 & 0.387 \\
\hline SMF-predictive & 0.946 & 4.123 & 0.570 & 0.899 & 3.439 & 0.444 \\
\hline
\end{tabular}

Table 1: Model 1: $X_{t+1}=\sin \left(X_{t}\right)+\epsilon_{t+1}$ with normal innovations. 


\begin{tabular}{|c|c|c|c|c|c|c|}
\hline Laplace innovations & \multicolumn{3}{|c|}{ nominal coverage $95 \%$} & \multicolumn{3}{|c|}{ nominal coverage $90 \%$} \\
\hline$n=50$ & CVR & LEN & st.err & CVR & LEN & st.err \\
\hline nonpara-f & 0.912 & 4.103 & 0.885 & 0.854 & 3.187 & 0.612 \\
\hline nonpara-p & 0.935 & 4.504 & 0.890 & 0.888 & 3.561 & 0.641 \\
\hline trans-forward & 0.913 & 4.072 & 1.033 & 0.873 & 3.429 & 0.894 \\
\hline trans-backward & 0.913 & 4.081 & 1.021 & 0.873 & 3.441 & 0.897 \\
\hline lbs-forward & 0.902 & 4.036 & 1.138 & 0.856 & 3.313 & 0.935 \\
\hline lbs-backward & 0.905 & 4.046 & 1.103 & 0.861 & 3.324 & 0.847 \\
\hline Hybrid-trans-f & 0.905 & 4.070 & 0.925 & 0.848 & 3.174 & 0.633 \\
\hline Hybrid-trans-p & 0.926 & 4.425 & 0.931 & 0.878 & 3.507 & 0.635 \\
\hline Hybrid-lbs-f & 0.913 & 4.081 & 0.934 & 0.857 & 3.184 & 0.623 \\
\hline Hybrid-lbs-p & 0.929 & 4.447 & 0.973 & 0.882 & 3.498 & 0.655 \\
\hline MF-fitted & 0.889 & 3.708 & 0.986 & 0.844 & 3.086 & 0.803 \\
\hline MF-predictive & 0.922 & 4.278 & 1.129 & 0.876 & 3.494 & 0.993 \\
\hline SMF-fitted & 0.891 & 3.715 & 0.963 & 0.846 & 3.084 & 0.764 \\
\hline SMF-predictive & 0.930 & 4.442 & 1.126 & 0.887 & 3.620 & 0.972 \\
\hline$n=100$ & CVR & LEN & st.err & CVR & LEN & st.err \\
\hline nonpara-f & 0.933 & 4.161 & 0.648 & 0.879 & 3.218 & 0.452 \\
\hline nonpare-p & 0.944 & 4.430 & 0.658 & 0.896 & 3.445 & 0.470 \\
\hline trans-forward & 0.925 & 4.236 & 1.027 & 0.885 & 3.424 & 0.763 \\
\hline trans-backward & 0.926 & 4.239 & 1.024 & 0.885 & 3.437 & 0.764 \\
\hline lbs-forward & 0.923 & 4.153 & 0.935 & 0.878 & 3.323 & 0.714 \\
\hline lbs-backward & 0.923 & 4.189 & 0.986 & 0.879 & 3.356 & 0.724 \\
\hline Hybrid-trans-f & 0.925 & 4.056 & 0.702 & 0.872 & 3.174 & 0.495 \\
\hline Hybrid-trans-p & 0.939 & 4.370 & 0.748 & 0.891 & 3.418 & 0.513 \\
\hline Hybrid-lbs-f & 0.927 & 4.094 & 0.687 & 0.876 & 3.202 & 0.493 \\
\hline Hybrid-lbs-p & 0.938 & 4.310 & 0.731 & 0.891 & 3.400 & 0.512 \\
\hline MF-fitted & 0.911 & 3.884 & 0.895 & 0.864 & 3.124 & 0.643 \\
\hline MF-predictive & 0.935 & 4.426 & 1.001 & 0.891 & 3.510 & 0.765 \\
\hline SMF-fitted & 0.910 & 3.846 & 0.856 & 0.864 & 3.106 & 0.623 \\
\hline SMF-predictive & 0.941 & 4.544 & 0.965 & 0.896 & 3.542 & 0.738 \\
\hline$n=200$ & CVR & LEN & st.err & CVR & LEN & st.err \\
\hline nonpara-f & 0.937 & 4.122 & 0.460 & 0.885 & 3.198 & 0.329 \\
\hline nonpara-p & 0.943 & 4.275 & 0.455 & 0.895 & 3.341 & 0.341 \\
\hline trans-forward & 0.928 & 4.184 & 0.914 & 0.884 & 3.307 & 0.619 \\
\hline trans-backward & 0.929 & 4.202 & 0.904 & 0.883 & 3.299 & 0.619 \\
\hline lbs-forward & 0.928 & 4.140 & 0.838 & 0.883 & 3.274 & 0.586 \\
\hline lbs-backward & 0.929 & 4.142 & 0.850 & 0.884 & 3.298 & 0.610 \\
\hline Hybrid-trans-f & 0.931 & 4.041 & 0.509 & 0.880 & 3.172 & 0.388 \\
\hline Hybrid-trans-p & 0.939 & 4.221 & 0.544 & 0.891 & 3.307 & 0.385 \\
\hline Hybrid-lbs-f & 0.932 & 4.041 & 0.560 & 0.881 & 3.170 & 0.405 \\
\hline Hybrid-lbs-p & 0.940 & 4.204 & 0.552 & 0.892 & 3.302 & 0.395 \\
\hline MF-fitted & 0.922 & 3.926 & 0.711 & 0.873 & 3.119 & 0.514 \\
\hline MF-predictive & 0.938 & 4.387 & 0.900 & 0.891 & 3.386 & 0.639 \\
\hline SMF-fitted & 0.921 & 3.895 & 0.684 & 0.873 & 3.103 & 0.491 \\
\hline SMF-predictive & 0.942 & 4.447 & 0.868 & 0.894 & 3.407 & 0.629 \\
\hline
\end{tabular}

Table 2: Model 1: $X_{t+1}=\sin \left(X_{t}\right)+\epsilon_{t+1}$ with Laplace innovations. 


\begin{tabular}{|c|c|c|c|c|c|c|}
\hline Normal innovations & nominal & cover & e $95 \%$ & nominal & \multicolumn{2}{|c|}{ coverage $90 \%$} \\
\hline$n=50$ & CVR & LEN & st.err & CVR & LEN & st.err \\
\hline nonpara-f & 0.907 & 3.873 & 0.558 & 0.850 & 3.256 & 0.456 \\
\hline nonpara-p & 0.937 & 4.289 & 0.570 & 0.891 & 3.615 & 0.452 \\
\hline trans-forward & 0.936 & 4.270 & 0.798 & 0.893 & 3.658 & 0.720 \\
\hline trans-backward & 0.937 & 4.278 & 0.812 & 0.893 & 3.667 & 0.723 \\
\hline lbs-forward & 0.908 & 3.982 & 0.869 & 0.862 & 3.426 & 0.761 \\
\hline lbs-backward & 0.912 & 4.016 & 0.863 & 0.865 & 3.446 & 0.747 \\
\hline Hybrid-trans-f & 0.901 & 3.862 & 0.557 & 0.845 & 3.265 & 0.464 \\
\hline Hybrid-trans-p & 0.928 & 4.255 & 0.625 & 0.879 & 3.600 & 0.507 \\
\hline Hybrid-lbs-f & 0.916 & 3.836 & 0.537 & 0.861 & 3.231 & 0.448 \\
\hline Hybrid-lbs-p & 0.940 & 4.231 & 0.622 & 0.893 & 3.562 & 0.486 \\
\hline MF-fitted & 0.894 & 3.763 & 0.850 & 0.843 & 3.228 & 0.750 \\
\hline MF-predictive & 0.932 & 4.269 & 0.927 & 0.886 & 3.614 & 0.794 \\
\hline SMF-fitted & 0.893 & 3.713 & 0.805 & 0.844 & 3.211 & 0.713 \\
\hline SMF-predictive & 0.943 & 4.440 & 0.885 & 0.902 & 3.755 & 0.751 \\
\hline$n=100$ & CVR & LEN & st.err & CVR & LEN & st.err \\
\hline nonpara-f & 0.928 & 3.870 & 0.388 & 0.875 & 3.260 & 0.308 \\
\hline nonpara-p & 0.946 & 4.143 & 0.393 & 0.900 & 3.495 & 0.305 \\
\hline trans-forward & 0.948 & 4.219 & 0.608 & 0.906 & 3.600 & 0.526 \\
\hline trans-backward & 0.948 & 4.225 & 0.612 & 0.905 & 3.594 & 0.525 \\
\hline lbs-forward & 0.932 & 4.016 & 0.642 & 0.887 & 3.437 & 0.557 \\
\hline lbs-backward & 0.932 & 4.031 & 0.648 & 0.888 & 3.445 & 0.574 \\
\hline Hybrid-trans-f & 0.921 & 3.828 & 0.437 & 0.867 & 3.238 & 0.352 \\
\hline Hybrid-trans-p & 0.940 & 4.089 & 0.435 & 0.894 & 3.476 & 0.345 \\
\hline Hybrid-lbs-f & 0.926 & 3.824 & 0.412 & 0.874 & 3.242 & 0.334 \\
\hline Hybrid-lbs-p & 0.943 & 4.073 & 0.439 & 0.895 & 3.438 & 0.327 \\
\hline MF-fitted & 0.918 & 3.790 & 0.620 & 0.868 & 3.247 & 0.542 \\
\hline MF-predictive & 0.945 & 4.218 & 0.706 & 0.901 & 3.560 & 0.569 \\
\hline SMF-fitted & 0.915 & 3.733 & 0.597 & 0.865 & 3.202 & 0.516 \\
\hline SMF-predictive & 0.950 & 4.315 & 0.695 & 0.906 & 3.600 & 0.550 \\
\hline$n=200$ & CVR & LEN & st.err & CVR & LEN & st.err \\
\hline nonpara-f & 0.936 & 3.877 & 0.297 & 0.883 & 3.270 & 0.238 \\
\hline nonpara-p & 0.948 & 4.053 & 0.295 & 0.899 & 3.418 & 0.238 \\
\hline trans-forward & 0.949 & 4.151 & 0.512 & 0.907 & 3.533 & 0.448 \\
\hline trans-backward & 0.948 & 4.147 & 0.529 & 0.907 & 3.530 & 0.446 \\
\hline lbs-forward & 0.936 & 4.008 & 0.585 & 0.890 & 3.387 & 0.473 \\
\hline lbs-backward & 0.936 & 3.993 & 0.559 & 0.891 & 3.387 & 0.470 \\
\hline Hybrid-trans-f & 0.931 & 3.841 & 0.354 & 0.880 & 3.253 & 0.290 \\
\hline Hybrid-trans-p & 0.943 & 4.022 & 0.360 & 0.895 & 3.399 & 0.289 \\
\hline Hybrid-lbs-f & 0.930 & 3.815 & 0.342 & 0.879 & 3.237 & 0.276 \\
\hline Hybrid-lbs-p & 0.942 & 3.997 & 0.374 & 0.895 & 3.390 & 0.296 \\
\hline MF-fitted & 0.924 & 3.773 & 0.524 & 0.875 & 3.212 & 0.431 \\
\hline MF-predictive & 0.944 & 4.126 & 0.609 & 0.895 & 3.419 & 0.472 \\
\hline SMF-fitted & 0.921 & 3.725 & 0.495 & 0.873 & 3.195 & 0.417 \\
\hline SMF-predictive & 0.947 & 4.163 & 0.605 & 0.899 & 3.447 & 0.469 \\
\hline
\end{tabular}

Table 3: Model 2: $X_{t+1}=0.8 \log \left(3 X_{t}^{2}+1\right)+\epsilon_{t+1}$ with normal innovations. 


\begin{tabular}{|c|c|c|c|c|c|c|}
\hline Laplace innovations & nominal & cover & e $95 \%$ & nominal & covera & e $90 \%$ \\
\hline$n=50$ & CVR & LEN & st.err & CVR & LEN & st.err \\
\hline nonpara-f & 0.912 & 4.111 & 0.884 & 0.853 & 3.205 & 0.606 \\
\hline nonpara-p & 0.938 & 4.598 & 0.894 & 0.890 & 3.628 & 0.633 \\
\hline trans-forward & 0.919 & 4.252 & 1.166 & 0.880 & 3.565 & 0.991 \\
\hline trans-backward & 0.919 & 4.250 & 1.153 & 0.879 & 3.571 & 1.014 \\
\hline lbs-forward & 0.895 & 4.021 & 1.263 & 0.854 & 3.360 & 1.021 \\
\hline lbs-backward & 0.900 & 4.004 & 1.188 & 0.856 & 3.339 & 0.982 \\
\hline Hybrid-trans-f & 0.909 & 4.068 & 0.878 & 0.848 & 3.201 & 0.635 \\
\hline Hybrid-trans-p & 0.932 & 4.555 & 0.907 & 0.884 & 3.599 & 0.633 \\
\hline Hybrid-lbs-f & 0.913 & 4.045 & 0.923 & 0.859 & 3.175 & 0.617 \\
\hline Hybrid-lbs-p & 0.934 & 4.489 & 0.960 & 0.887 & 3.524 & 0.648 \\
\hline MF-fitted & 0.886 & 3.774 & 1.094 & 0.840 & 3.145 & 0.918 \\
\hline MF-predictive & 0.924 & 4.412 & 1.222 & 0.881 & 3.587 & 1.035 \\
\hline SMF-fitted & 0.886 & 3.744 & 1.047 & 0.841 & 3.117 & 0.855 \\
\hline SMF-predictive & 0.933 & 4.599 & 1.209 & 0.890 & 3.707 & 1.022 \\
\hline$n=100$ & CVR & LEN & st.err & CVR & LEN & st.err \\
\hline nonpara-f & 0.932 & 4.175 & 0.639 & 0.880 & 3.237 & 0.449 \\
\hline nonpara-p & 0.944 & 4.458 & 0.649 & 0.898 & 3.472 & 0.464 \\
\hline trans-forward & 0.932 & 4.372 & 1.071 & 0.893 & 3.549 & 0.797 \\
\hline trans-backward & 0.933 & 4.396 & 1.080 & 0.895 & 3.573 & 0.799 \\
\hline lbs-forward & 0.920 & 4.179 & 1.041 & 0.877 & 3.375 & 0.813 \\
\hline lbs-backward & 0.922 & 4.239 & 1.081 & 0.880 & 3.417 & 0.830 \\
\hline Hybrid-trans-f & 0.925 & 4.101 & 0.685 & 0.872 & 3.198 & 0.510 \\
\hline Hybrid-trans-p & 0.938 & 4.410 & 0.731 & 0.891 & 3.430 & 0.515 \\
\hline Hybrid-lbs-f & 0.929 & 4.092 & 0.683 & 0.877 & 3.205 & 0.497 \\
\hline Hybrid-lbs-p & 0.940 & 4.359 & 0.718 & 0.895 & 3.426 & 0.526 \\
\hline MF-fitted & 0.908 & 3.904 & 0.961 & 0.863 & 3.162 & 0.744 \\
\hline MF-predictive & 0.937 & 4.581 & 1.100 & 0.893 & 3.589 & 0.881 \\
\hline SMF-fitted & 0.909 & 3.848 & 0.882 & 0.863 & 3.128 & 0.683 \\
\hline SMF-predictive & 0.941 & 4.637 & 1.060 & 0.898 & 3.630 & 0.822 \\
\hline$n=200$ & CVR & LEN & st.err & CVR & LEN & st.err \\
\hline nonpara-f & 0.937 & 4.110 & 0.455 & 0.884 & 3.192 & 0.322 \\
\hline nonpara-p & 0.944 & 4.284 & 0.452 & 0.895 & 3.347 & 0.336 \\
\hline trans-forward & 0.935 & 4.336 & 0.943 & 0.893 & 3.454 & 0.664 \\
\hline trans-backward & 0.935 & 4.337 & 0.963 & 0.893 & 3.454 & 0.674 \\
\hline lbs-forward & 0.931 & 4.259 & 0.947 & 0.886 & 3.385 & 0.740 \\
\hline lbs-backward & 0.930 & 4.228 & 0.929 & 0.883 & 3.346 & 0.663 \\
\hline Hybrid-trans-f & 0.930 & 4.021 & 0.522 & 0.878 & 3.158 & 0.387 \\
\hline Hybrid-trans-p & 0.938 & 4.186 & 0.526 & 0.890 & 3.315 & 0.397 \\
\hline Hybrid-lbs-f & 0.931 & 4.016 & 0.564 & 0.880 & 3.151 & 0.393 \\
\hline Hybrid-lbs-p & 0.940 & 4.209 & 0.550 & 0.893 & 3.310 & 0.378 \\
\hline MF-fitted & 0.922 & 3.974 & 0.793 & 0.872 & 3.145 & 0.560 \\
\hline MF-predictive & 0.939 & 4.464 & 1.000 & 0.893 & 3.444 & 0.701 \\
\hline SMF-fitted & 0.920 & 3.941 & 0.749 & 0.872 & 3.123 & 0.540 \\
\hline SMF-predictive & 0.941 & 4.497 & 0.990 & 0.895 & 3.471 & 0.689 \\
\hline
\end{tabular}

Table 4: Model 2: $X_{t+1}=0.8 \log \left(3 X_{t}^{2}+1\right)+\epsilon_{t+1}$ with Laplace innovations. 


\begin{tabular}{|c|c|c|c|c|c|c|}
\hline Normal innovations & \multicolumn{3}{|c|}{ nominal coverage $95 \%$} & \multicolumn{3}{|c|}{ nominal coverage $90 \%$} \\
\hline$n=50$ & CVR & LEN & st.err & CVR & LEN & st.err \\
\hline nonpara-f & 0.915 & 3.757 & 0.519 & 0.864 & 3.192 & 0.415 \\
\hline nonpara-p & 0.933 & 3.973 & 0.503 & 0.886 & 3.378 & 0.390 \\
\hline trans-forward & 0.910 & 3.821 & 0.716 & 0.862 & 3.310 & 0.638 \\
\hline trans-backward & 0.911 & 3.840 & 0.706 & 0.862 & 3.316 & 0.628 \\
\hline lbs-forward & 0.912 & 3.744 & 0.648 & 0.862 & 3.200 & 0.534 \\
\hline lbs-backward & 0.913 & 3.771 & 0.649 & 0.863 & 3.220 & 0.525 \\
\hline Hybrid-trans-f & 0.912 & 3.749 & 0.538 & 0.860 & 3.169 & 0.415 \\
\hline Hybrid-trans-p & 0.926 & 3.918 & 0.521 & 0.880 & 3.350 & 0.430 \\
\hline Hybrid-lbs-f & 0.913 & 3.721 & 0.524 & 0.863 & 3.172 & 0.418 \\
\hline Hybrid-lbs-p & 0.929 & 3.935 & 0.531 & 0.882 & 3.334 & 0.424 \\
\hline MF-fitted & 0.893 & 3.570 & 0.651 & 0.844 & 3.100 & 0.580 \\
\hline MF-predictive & 0.922 & 3.892 & 0.697 & 0.874 & 3.338 & 0.588 \\
\hline SMF-fitted & 0.893 & 3.566 & 0.643 & 0.843 & 3.084 & 0.567 \\
\hline SMF-predictive & 0.935 & 4.057 & 0.676 & 0.889 & 3.477 & 0.589 \\
\hline$n=100$ & CVR & LEN & st.err & CVR & LEN & st.err \\
\hline nonpara-f & 0.932 & 3.832 & 0.369 & 0.881 & 3.236 & 0.282 \\
\hline nonpara-p & 0.940 & 3.946 & 0.380 & 0.892 & 3.332 & 0.296 \\
\hline trans-forward & 0.929 & 3.933 & 0.588 & 0.883 & 3.373 & 0.506 \\
\hline trans-backward & 0.928 & 3.934 & 0.589 & 0.882 & 3.366 & 0.505 \\
\hline lbs-forward & 0.927 & 3.818 & 0.499 & 0.879 & 3.248 & 0.399 \\
\hline lbs-backward & 0.928 & 3.828 & 0.487 & 0.879 & 3.256 & 0.391 \\
\hline Hybrid-trans-f & 0.925 & 3.769 & 0.415 & 0.874 & 3.194 & 0.324 \\
\hline Hybrid-trans-p & 0.934 & 3.882 & 0.421 & 0.886 & 3.300 & 0.331 \\
\hline Hybrid-lbs-f & 0.928 & 3.798 & 0.419 & 0.877 & 3.217 & 0.324 \\
\hline Hybrid-lbs-p & 0.935 & 3.880 & 0.412 & 0.887 & 3.294 & 0.314 \\
\hline MF-fitted & 0.919 & 3.736 & 0.506 & 0.870 & 3.195 & 0.410 \\
\hline MF-predictive & 0.937 & 3.996 & 0.571 & 0.888 & 3.360 & 0.443 \\
\hline SMF-fitted & 0.920 & 3.711 & 0.465 & 0.871 & 3.184 & 0.394 \\
\hline SMF-predictive & 0.944 & 4.092 & 0.551 & 0.898 & 3.439 & 0.433 \\
\hline$n=200$ & CVR & LEN & st.err & CVR & LEN & st.err \\
\hline nonpara-f & 0.941 & 3.862 & 0.265 & 0.890 & 3.257 & 0.210 \\
\hline nonpara-p & 0.945 & 3.926 & 0.274 & 0.896 & 3.312 & 0.214 \\
\hline trans-forward & 0.934 & 3.938 & 0.528 & 0.888 & 3.345 & 0.442 \\
\hline trans-backward & 0.934 & 3.938 & 0.537 & 0.887 & 3.339 & 0.443 \\
\hline lbs-forward & 0.935 & 3.846 & 0.404 & 0.885 & 3.246 & 0.304 \\
\hline lbs-backward & 0.934 & 3.832 & 0.386 & 0.885 & 3.253 & 0.308 \\
\hline Hybrid-trans-f & 0.936 & 3.818 & 0.321 & 0.886 & 3.238 & 0.263 \\
\hline Hybrid-trans-p & 0.940 & 3.879 & 0.342 & 0.892 & 3.293 & 0.265 \\
\hline Hybrid-lbs-f & 0.934 & 3.790 & 0.332 & 0.885 & 3.226 & 0.268 \\
\hline Hybrid-lbs-p & 0.940 & 3.882 & 0.337 & 0.892 & 3.294 & 0.267 \\
\hline MF-fitted & 0.930 & 3.783 & 0.415 & 0.882 & 3.224 & 0.326 \\
\hline MF-predictive & 0.940 & 3.946 & 0.470 & 0.893 & 3.329 & 0.358 \\
\hline SMF-fitted & 0.931 & 3.779 & 0.391 & 0.882 & 3.218 & 0.311 \\
\hline SMF-predictive & 0.945 & 4.019 & 0.451 & 0.898 & 3.377 & 0.349 \\
\hline
\end{tabular}

Table 5: Model 3: $X_{t+1}=-0.5 \exp \left(-50 X_{t}^{2}\right) X_{t}+\epsilon_{t+1}$ with normal innovations. 


\begin{tabular}{|c|c|c|c|c|c|c|}
\hline Laplace innovations & nominal & cover & e $95 \%$ & nomina & cover & se $90 \%$ \\
\hline$n=50$ & CVR & LEN & st.err & CVR & LEN & st.err \\
\hline nonpara-f & 0.922 & 4.110 & 0.858 & 0.867 & 3.176 & 0.586 \\
\hline nonpara-p & 0.932 & 4.300 & 0.861 & 0.885 & 3.390 & 0.616 \\
\hline trans-forward & 0.881 & 3.791 & 1.207 & 0.835 & 3.186 & 1.032 \\
\hline trans-backward & 0.884 & 3.827 & 1.225 & 0.838 & 3.208 & 1.033 \\
\hline lbs-forward & 0.910 & 4.090 & 1.178 & 0.858 & 3.182 & 0.788 \\
\hline lbs-backward & 0.910 & 4.035 & 1.103 & 0.858 & 3.172 & 0.766 \\
\hline Hybrid-trans-f & 0.917 & 4.058 & 0.904 & 0.862 & 3.156 & 0.661 \\
\hline Hybrid-trans-p & 0.930 & 4.312 & 1.040 & 0.881 & 3.367 & 0.643 \\
\hline Hybrid-lbs-f & 0.917 & 4.041 & 0.922 & 0.866 & 3.143 & 0.605 \\
\hline Hybrid-lbs-p & 0.290 & 4.263 & 0.971 & 0.880 & 3.298 & 0.615 \\
\hline MF-fitted & 0.888 & 3.665 & 0.933 & 0.840 & 3.011 & 0.754 \\
\hline MF-predictive & 0.916 & 4.146 & 1.082 & 0.874 & 3.401 & 0.864 \\
\hline SMF-fitted & 0.892 & 3.686 & 0.902 & 0.844 & 3.021 & 0.715 \\
\hline SMF-predictive & 0.926 & 4.270 & 1.015 & 0.883 & 3.482 & 0.847 \\
\hline$n=100$ & CVR & LEN & st.err & CVR & LEN & st.err \\
\hline nonpara-f & 0.934 & 4.192 & 0.636 & 0.882 & 3.228 & 0.447 \\
\hline nonpara-p & 0.938 & 4.295 & 0.640 & 0.890 & 3.322 & 0.455 \\
\hline trans-forward & 0.911 & 4.102 & 1.114 & 0.867 & 3.336 & 0.864 \\
\hline trans-backward & 0.912 & 4.109 & 1.110 & 0.867 & 3.341 & 0.865 \\
\hline lbs-forward & 0.929 & 4.162 & 0.819 & 0.878 & 3.229 & 0.597 \\
\hline lbs-backward & 0.928 & 4.156 & 0.822 & 0.881 & 3.261 & 0.597 \\
\hline Hybrid-trans-f & 0.925 & 4.050 & 0.682 & 0.875 & 3.176 & 0.508 \\
\hline Hybrid-trans-p & 0.932 & 4.231 & 0.760 & 0.883 & 3.294 & 0.519 \\
\hline Hybrid-lbs-f & 0.928 & 4.090 & 0.677 & 0.877 & 3.189 & 0.502 \\
\hline Hybrid-lbs-p & 0.934 & 4.215 & 0.706 & 0.885 & 3.281 & 0.498 \\
\hline MF-fitted & 0.920 & 3.961 & 0.746 & 0.871 & 3.149 & 0.559 \\
\hline MF-predictive & 0.938 & 4.321 & 0.936 & 0.893 & 3.450 & 0.657 \\
\hline SMF-fitted & 0.921 & 3.949 & 0.711 & 0.872 & 3.130 & 0.514 \\
\hline SMF-predictive & 0.943 & 4.499 & 0.857 & 0.897 & 3.501 & 0.626 \\
\hline$n=200$ & CVR & LEN & st.err & CVR & LEN & st.err \\
\hline nonpara-f & 0.939 & 4.143 & 0.478 & 0.889 & 3.205 & 0.327 \\
\hline nonpara-p & 0.941 & 4.190 & 0.467 & 0.893 & 3.269 & 0.337 \\
\hline trans-forward & 0.916 & 4.060 & 1.038 & 0.870 & 3.224 & 0.739 \\
\hline trans-backward & 0.917 & 4.069 & 1.040 & 0.871 & 3.227 & 0.726 \\
\hline lbs-forward & 0.931 & 4.055 & 0.658 & 0.881 & 3.161 & 0.449 \\
\hline lbs-backward & 0.932 & 4.081 & 0.647 & 0.883 & 3.203 & 0.467 \\
\hline Hybrid-trans-f & 0.935 & 4.081 & 0.539 & 0.885 & 3.171 & 0.372 \\
\hline Hybrid-trans-p & 0.936 & 4.100 & 0.580 & 0.888 & 3.224 & 0.397 \\
\hline Hybrid-lbs-f & 0.934 & 4.057 & 0.559 & 0.883 & 3.168 & 0.413 \\
\hline Hybrid-lbs-p & 0.937 & 4.128 & 0.559 & 0.888 & 3.231 & 0.401 \\
\hline MF-fitted & 0.927 & 3.991 & 0.701 & 0.879 & 3.153 & 0.504 \\
\hline MF-predictive & 0.938 & 4.331 & 0.894 & 0.890 & 3.318 & 0.568 \\
\hline SMF-fitted & 0.927 & 3.962 & 0.651 & 0.879 & 3.139 & 0.476 \\
\hline SMF-predictive & 0.942 & 4.403 & 0.863 & 0.892 & 3.340 & 0.551 \\
\hline
\end{tabular}

Table 6: Model 3: $X_{t+1}=-0.5 \exp \left(-50 X_{t}^{2}\right) X_{t}+\epsilon_{t+1}$ with Laplace innovations. 


\begin{tabular}{|c|c|c|c|c|c|c|}
\hline Normal innovations & nominal & cover & e $95 \%$ & nominal & \multicolumn{2}{|c|}{ coverage $90 \%$} \\
\hline$n=50$ & CVR & LEN & st.err & CVR & LEN & st.err \\
\hline nonpara-f & 0.864 & 3.112 & 1.276 & 0.813 & 2.671 & 1.089 \\
\hline nonpara-p & 0.904 & 3.645 & 1.538 & 0.850 & 2.967 & 1.218 \\
\hline trans-forward & 0.927 & 3.477 & 0.802 & 0.882 & 2.983 & 0.716 \\
\hline trans-backward & 0.928 & 3.495 & 0.832 & 0.882 & 2.992 & 0.722 \\
\hline lbs-forward & 0.914 & 3.424 & 0.833 & 0.867 & 2.893 & 0.717 \\
\hline lbs-backward & 0.914 & 3.451 & 0.803 & 0.867 & 2.924 & 0.692 \\
\hline Hybrid-trans-f & 0.865 & 3.169 & 1.212 & 0.812 & 2.721 & 1.050 \\
\hline Hybrid-trans-p & 0.897 & 3.639 & 1.494 & 0.840 & 2.978 & 1.148 \\
\hline Hybrid-lbs-f & 0.862 & 3.146 & 1.256 & 0.808 & 2.686 & 1.070 \\
\hline Hybrid-lbs-p & 0.895 & 3.639 & 1.637 & 0.840 & 2.956 & 1.180 \\
\hline MF-fitted & 0.891 & 3.084 & 0.742 & 0.841 & 2.660 & 0.670 \\
\hline MF-predictive & 0.926 & 3.549 & 0.849 & 0.880 & 2.989 & 0.725 \\
\hline SMF-fitted & 0.890 & 3.073 & 0.712 & 0.842 & 2.653 & 0.630 \\
\hline SMF-predictive & 0.931 & 3.687 & 0.836 & 0.891 & 3.078 & 0.699 \\
\hline$n=100$ & CVR & LEN & st.err & CVR & LEN & st.err \\
\hline nonpara-f & 0.894 & 3.015 & 0.926 & 0.843 & 2.566 & 0.783 \\
\hline nonpara-p & 0.922 & 3.318 & 1.003 & 0.868 & 2.744 & 0.826 \\
\hline trans-forward & 0.943 & 3.421 & 0.629 & 0.901 & 2.928 & 0.561 \\
\hline trans-backward & 0.943 & 3.439 & 0.648 & 0.901 & 2.930 & 0.573 \\
\hline lbs-forward & 0.938 & 3.425 & 0.628 & 0.895 & 2.908 & 0.553 \\
\hline lbs-backward & 0.937 & 3.410 & 0.616 & 0.894 & 2.903 & 0.549 \\
\hline Hybrid-trans-f & 0.888 & 2.997 & 0.873 & 0.833 & 2.564 & 0.747 \\
\hline Hybrid-trans-p & 0.914 & 3.301 & 0.980 & 0.855 & 3.726 & 0.792 \\
\hline Hybrid-lbs-f & 0.888 & 2.988 & 0.877 & 0.834 & 2.553 & 0.748 \\
\hline Hybrid-lbs-p & 0.916 & 3.301 & 0.996 & 0.858 & 2.727 & 0.796 \\
\hline MF-fitted & 0.921 & 3.134 & 0.578 & 0.874 & 2.685 & 0.504 \\
\hline MF-predictive & 0.945 & 3.495 & 0.636 & 0.900 & 2.916 & 0.533 \\
\hline SMF-fitted & 0.921 & 3.119 & 0.551 & 0.874 & 2.679 & 0.476 \\
\hline SMF-predictive & 0.951 & 3.587 & 0.620 & 0.908 & 2.964 & 0.513 \\
\hline$n=200$ & CVR & LEN & st.err & CVR & LEN & st.err \\
\hline nonpara-f & 0.903 & 2.903 & 0.774 & 0.848 & 2.537 & 0.647 \\
\hline nonpara-p & 0.921 & 3.164 & 0.789 & 0.863 & 2.636 & 0.654 \\
\hline trans-forward & 0.943 & 3.428 & 0.627 & 0.901 & 2.921 & 0.548 \\
\hline trans-backward & 0.943 & 3.430 & 0.633 & 0.901 & 2.921 & 0.552 \\
\hline lbs-forward & 0.942 & 3.425 & 0.578 & 0.898 & 2.894 & 0.483 \\
\hline lbs-backward & 0.941 & 3.406 & 0.562 & 0.895 & 2.858 & 0.462 \\
\hline Hybrid-trans-f & 0.892 & 2.991 & 0.789 & 0.836 & 2.541 & 0.652 \\
\hline Hybrid-trans-p & 0.908 & 3.147 & 0.816 & 0.849 & 2.631 & 0.663 \\
\hline Hybrid-lbs-f & 0.892 & 2.953 & 0.763 & 0.837 & 2.520 & 0.643 \\
\hline Hybrid-lbs-p & 0.911 & 3.148 & 0.810 & 0.853 & 2.628 & 0.663 \\
\hline MF-fitted & 0.927 & 3.192 & 0.539 & 0.880 & 2.716 & 0.439 \\
\hline MF-predictive & 0.944 & 3.458 & 0.636 & 0.897 & 2.866 & 0.476 \\
\hline SMF-fitted & 0.926 & 3.167 & 0.504 & 0.879 & 2.707 & 0.420 \\
\hline SMF-predictive & 0.947 & 3.481 & 0.574 & 0.900 & 2.890 & 0.473 \\
\hline
\end{tabular}

Table 7: Model 4: $X_{t+1}=\sin \left(X_{t}\right)+\sqrt{0.5+0.25 X_{t}^{2}} \epsilon_{t+1}$ with normal innovations. 


\begin{tabular}{|c|c|c|c|c|c|c|}
\hline Laplace innovations & \multicolumn{3}{|c|}{ nominal coverage $95 \%$} & \multicolumn{3}{|c|}{ nominal coverage $90 \%$} \\
\hline$n=50$ & CVR & LEN & st.err & CVR & LEN & st.err \\
\hline nonpara-f & 0.862 & 3.173 & 1.676 & 0.811 & 2.532 & 1.230 \\
\hline nonpara-p & 0.904 & 3.881 & 2.078 & 0.849 & 2.878 & 1.354 \\
\hline trans-forward & 0.910 & 3.359 & 0.964 & 0.870 & 2.828 & 0.847 \\
\hline trans-backward & 0.911 & 3.369 & 0.953 & 0.871 & 2.839 & 0.841 \\
\hline lbs-forward & 0.904 & 3.423 & 1.029 & 0.864 & 2.809 & 0.818 \\
\hline lbs-backward & 0.907 & 3.433 & 0.983 & 0.864 & 2.798 & 0.766 \\
\hline Hybrid-trans-f & 0.866 & 3.209 & 1.562 & 0.814 & 2.595 & 1.195 \\
\hline Hybrid-trans-p & 0.899 & 3.848 & 1.980 & 0.841 & 2.895 & 1.340 \\
\hline Hybrid-lbs-f & 0.866 & 3.194 & 1.674 & 0.814 & 2.572 & 1.245 \\
\hline Hybrid-lbs-p & 0.901 & 3.875 & 2.093 & 0.846 & 2.894 & 1.391 \\
\hline MF-fitted & 0.891 & 3.102 & 0.932 & 0.846 & 2.554 & 0.764 \\
\hline MF-predictive & 0.920 & 3.616 & 1.064 & 0.879 & 2.916 & 0.855 \\
\hline SMF-fitted & 0.893 & 3.093 & 0.871 & 0.846 & 2.539 & 0.707 \\
\hline SMF-predictive & 0.930 & 3.774 & 1.094 & 0.888 & 3.017 & 0.857 \\
\hline$n=100$ & CVR & LEN & st.err & CVR & LEN & st.err \\
\hline nonpara-f & 0.895 & 3.197 & 1.270 & 0.843 & 2.521 & 0.909 \\
\hline nonpara-p & 0.921 & 3.662 & 1.515 & 0.866 & 2.740 & 0.967 \\
\hline trans-forward & 0.926 & 3.518 & 0.952 & 0.887 & 2.867 & 0.779 \\
\hline trans-backward & 0.926 & 3.526 & 0.963 & 0.886 & 2.872 & 0.789 \\
\hline lbs-forward & 0.924 & 3.482 & 0.839 & 0.877 & 2.762 & 0.654 \\
\hline lbs-backward & 0.924 & 3.531 & 0.871 & 0.881 & 2.810 & 0.676 \\
\hline Hybrid-trans-f & 0.885 & 3.149 & 1.232 & 0.830 & 2.499 & 0.890 \\
\hline Hybrid-trans-p & 0.910 & 3.576 & 1.468 & 0.849 & 2.707 & 0.990 \\
\hline Hybrid-lbs-f & 0.888 & 3.153 & 1.201 & 0.835 & 2.514 & 0.906 \\
\hline Hybrid-lbs-p & 0.913 & 3.580 & 1.470 & 0.854 & 2.699 & 1.026 \\
\hline MF-fitted & 0.912 & 3.200 & 0.740 & 0.865 & 2.578 & 0.581 \\
\hline MF-predictive & 0.938 & 3.755 & 0.905 & 0.894 & 2.918 & 0.663 \\
\hline SMF-fitted & 0.912 & 3.177 & 0.724 & 0.864 & 2.559 & 0.555 \\
\hline SMF-predictive & 0.943 & 3.880 & 0.892 & 0.899 & 2.950 & 0.605 \\
\hline$n=200$ & CVR & LEN & st.err & CVR & LEN & st.err \\
\hline nonpara-f & 0.905 & 3.028 & 0.955 & 0.851 & 2.395 & 0.747 \\
\hline nonpara-p & 0.921 & 3.285 & 1.029 & 0.864 & 2.514 & 0.776 \\
\hline trans-forward & 0.932 & 3.492 & 0.915 & 0.890 & 2.783 & 0.714 \\
\hline trans-backward & 0.932 & 3.494 & 0.920 & 0.890 & 2.780 & 0.721 \\
\hline lbs-forward & 0.932 & 3.425 & 0.717 & 0.888 & 2.724 & 0.580 \\
\hline lbs-backward & 0.933 & 3.477 & 0.735 & 0.888 & 2.751 & 0.592 \\
\hline Hybrid-trans-f & 0.894 & 2.998 & 1.006 & 0.837 & 2.386 & 0.768 \\
\hline Hybrid-trans-p & 0.910 & 3.241 & 1.040 & 0.850 & 2.494 & 0.768 \\
\hline Hybrid-lbs-f & 0.897 & 3.006 & 0.961 & 0.841 & 2.397 & 0.765 \\
\hline Hybrid-lbs-p & 0.911 & 3.229 & 1.036 & 0.852 & 2.481 & 0.752 \\
\hline MF-fitted & 0.926 & 3.261 & 0.695 & 0.878 & 2.572 & 0.502 \\
\hline MF-predictive & 0.942 & 3.648 & 0.862 & 0.895 & 2.774 & 0.664 \\
\hline SMF-fitted & 0.926 & 3.224 & 0.648 & 0.878 & 2.559 & 0.463 \\
\hline SMF-predictive & 0.945 & 3.716 & 0.846 & 0.898 & 2.812 & 0.562 \\
\hline
\end{tabular}

Table 8: Model 4: $X_{t+1}=\sin \left(X_{t}\right)+\sqrt{0.5+0.25 X_{t}^{2}} \epsilon_{t+1}$ with Laplace innovations. 


\begin{tabular}{|c|c|c|c|c|c|c|}
\hline Normal innovations & \multicolumn{3}{|c|}{ nominal coverage $95 \%$} & \multicolumn{3}{|c|}{ nominal coverage $90 \%$} \\
\hline$n=50$ & CVR & LEN & st.err & CVR & LEN & st.err \\
\hline nonpara-f & 0.897 & 4.067 & 1.596 & 0.854 & 3.493 & 1.365 \\
\hline nonpara-p & 0.931 & 4.669 & 1.820 & 0.887 & 3.856 & 1.479 \\
\hline trans-forward & 0.941 & 4.286 & 0.987 & 0.902 & 3.670 & 0.870 \\
\hline trans-backward & 0.940 & 4.284 & 0.993 & 0.901 & 3.666 & 0.870 \\
\hline lbs-forward & 0.914 & 3.967 & 1.039 & 0.870 & 3.407 & 0.907 \\
\hline lbs-backward & 0.916 & 6.993 & 0.991 & 0.871 & 3.406 & 0.879 \\
\hline Hybrid-trans-f & 0.904 & 4.121 & 1.467 & 0.860 & 3.552 & 1.289 \\
\hline Hybrid-trans-p & 0.931 & 4.683 & 1.720 & 0.887 & 3.891 & 1.392 \\
\hline Hybrid-lbs-f & 0.901 & 4.079 & 1.516 & 0.857 & 3.508 & 1.306 \\
\hline Hybrid-lbs-p & 0.929 & 4.636 & 1.756 & 0.884 & 3.832 & 1.403 \\
\hline MF-fitted & 0.898 & 3.724 & 0.980 & 0.850 & 3.204 & 0.855 \\
\hline MF-predictive & 0.941 & 4.310 & 1.121 & 0.898 & 3.635 & 0.919 \\
\hline SMF-fitted & 0.898 & 3.710 & 0.937 & 0.852 & 3.206 & 0.812 \\
\hline SMF-predictive & 0.949 & 4.509 & 1.071 & 0.910 & 3.772 & 0.883 \\
\hline$n=100$ & CVR & LEN & st.err & CVR & LEN & st.err \\
\hline nonpara-f & 0.931 & 4.063 & 1.453 & 0.888 & 3.465 & 1.228 \\
\hline nonpara-p & 0.950 & 4.672 & 1.621 & 0.908 & 3.704 & 1.321 \\
\hline trans-forward & 0.950 & 4.203 & 0.885 & 0.913 & 3.584 & 0.747 \\
\hline trans-backward & 0.949 & 4.198 & 0.900 & 0.912 & 3.586 & 0.769 \\
\hline lbs-forward & 0.936 & 3.996 & 0.910 & 0.893 & 3.405 & 0.766 \\
\hline lbs-backward & 0.937 & 4.021 & 0.944 & 0.896 & 3.442 & 0.787 \\
\hline Hybrid-trans-f & 0.925 & 4.049 & 1.399 & 0.881 & 3.464 & 1.163 \\
\hline Hybrid-trans-p & 0.944 & 4.416 & 1.496 & 0.900 & 3.686 & 1.237 \\
\hline Hybrid-lbs-f & 0.924 & 4.005 & 1.393 & 0.881 & 3.437 & 1.186 \\
\hline Hybrid-lbs-p & 0.945 & 4.418 & 1.589 & 0.901 & 3.668 & 1.282 \\
\hline MF-fitted & 0.920 & 3.715 & 0.851 & 0.874 & 3.189 & 0.714 \\
\hline MF-predictive & 0.946 & 4.155 & 0.933 & 0.901 & 3.464 & 0.775 \\
\hline SMF-fitted & 0.920 & 3.689 & 0.794 & 0.874 & 3.177 & 0.667 \\
\hline SMF-predictive & 0.952 & 4.264 & 0.915 & 0.909 & 3.528 & 0.733 \\
\hline$n=200$ & CVR & LEN & st.err & CVR & LEN & st.err \\
\hline nonpara-f & 0.941 & 4.034 & 1.322 & 0.898 & 3.427 & 1.113 \\
\hline nonpara-p & 0.952 & 4.262 & 1.404 & 0.909 & 3.556 & 1.173 \\
\hline trans-forward & 0.954 & 4.109 & 0.815 & 0.916 & 3.502 & 0.689 \\
\hline trans-backward & 0.954 & 4.101 & 0.810 & 0.916 & 3.505 & 0.685 \\
\hline lbs-forward & 0.945 & 3.980 & 0.832 & 0.903 & 3.395 & 0.697 \\
\hline lbs-backward & 0.944 & 3.969 & 0.813 & 0.901 & 3.377 & 0.694 \\
\hline Hybrid-trans-f & 0.935 & 4.019 & 1.309 & 0.889 & 3.423 & 1.115 \\
\hline Hybrid-trans-p & 0.946 & 4.244 & 1.407 & 0.900 & 3.540 & 1.163 \\
\hline Hybrid-lbs-f & 0.932 & 3.971 & 1.293 & 0.886 & 3.382 & 1.098 \\
\hline Hybrid-lbs-p & 0.944 & 4.214 & 1.378 & 0.900 & 3.534 & 1.155 \\
\hline MF-fitted & 0.929 & 3.724 & 0.781 & 0.882 & 3.171 & 0.652 \\
\hline MF-predictive & 0.946 & 4.015 & 0.866 & 0.901 & 3.357 & 0.712 \\
\hline SMF-fitted & 0.928 & 3.700 & 0.731 & 0.882 & 3.165 & 0.624 \\
\hline SMF-predictive & 0.950 & 4.062 & 0.836 & 0.904 & 3.381 & 0.685 \\
\hline
\end{tabular}

Table 9: Model 5: $X_{t+1}=0.75 X_{t}+0.15 X_{t} \epsilon_{t+1}+\epsilon_{t+1}$ with normal innovations. 


\begin{tabular}{|c|c|c|c|c|c|c|}
\hline Laplace innovations & \multicolumn{3}{|c|}{ nominal coverage $95 \%$} & \multicolumn{3}{|c|}{ nominal coverage $90 \%$} \\
\hline$n=50$ & CVR & LEN & st.err & CVR & LEN & st.err \\
\hline nonpara-f & 0.901 & 4.399 & 2.441 & 0.858 & 3.559 & 1.932 \\
\hline nonpara-p & 0.932 & 5.427 & 3.144 & 0.889 & 4.082 & 2.197 \\
\hline trans-forward & 0.926 & 4.329 & 1.368 & 0.890 & 3.613 & 1.171 \\
\hline trans-backward & 0.925 & 4.304 & 1.353 & 0.889 & 3.599 & 1.164 \\
\hline lbs-forward & 0.905 & 4.144 & 1.410 & 0.864 & 3.429 & 1.168 \\
\hline lbs-backward & 0.910 & 4.177 & 1.371 & 0.869 & 3.454 & 1.178 \\
\hline Hybrid-trans-f & 0.905 & 4.441 & 2.343 & 0.864 & 3.603 & 1.798 \\
\hline Hybrid-trans-p & 0.931 & 5.352 & 2.875 & 0.889 & 4.099 & 2.040 \\
\hline Hybrid-lbs-f & 0.905 & 4.426 & 2.469 & 0.864 & 3.583 & 1.866 \\
\hline Hybrid-lbs-p & 0.932 & 5.423 & 3.217 & 0.889 & 4.073 & 2.223 \\
\hline MF-fitted & 0.892 & 3.837 & 1.327 & 0.846 & 3.166 & 1.138 \\
\hline MF-predictive & 0.926 & 4.435 & 1.482 & 0.882 & 3.575 & 1.224 \\
\hline SMF-fitted & 0.894 & 3.813 & 1.235 & 0.849 & 3.166 & 1.053 \\
\hline SMF-predictive & 0.937 & 4.748 & 1.497 & 0.898 & 3.757 & 1.149 \\
\hline$n=100$ & CVR & LEN & st.err & CVR & LEN & st.err \\
\hline nonpara-f & 0.923 & 4.414 & 2.283 & 0.881 & 3.512 & 1.811 \\
\hline nonpara-p & 0.943 & 5.081 & 2.865 & 0.899 & 3.827 & 2.001 \\
\hline trans-forward & 0.936 & 4.332 & 1.307 & 0.899 & 3.533 & 1.049 \\
\hline trans-backward & 0.936 & 4.330 & 1.258 & 0.899 & 3.531 & 1.026 \\
\hline lbs-forward & 0.926 & 4.196 & 1.269 & 0.883 & 3.364 & 1.014 \\
\hline lbs-backward & 0.926 & 4.173 & 1.240 & 0.885 & 3.385 & 1.045 \\
\hline Hybrid-trans-f & 0.920 & 4.351 & 2.149 & 0.876 & 3.501 & 1.700 \\
\hline Hybrid-trans-p & 0.938 & 5.020 & 3.051 & 0.893 & 3.805 & 1.988 \\
\hline Hybrid-lbs-f & 0.920 & 4.338 & 2.091 & 0.877 & 3.470 & 1.612 \\
\hline Hybrid-lbs-p & 0.938 & 4.976 & 2.835 & 0.893 & 3.759 & 1.941 \\
\hline MF-fitted & 0.911 & 3.822 & 1.167 & 0.865 & 3.085 & 0.898 \\
\hline MF-predictive & 0.941 & 4.564 & 1.436 & 0.896 & 3.507 & 1.054 \\
\hline SMF-fitted & 0.911 & 3.779 & 1.066 & 0.866 & 3.072 & 0.840 \\
\hline SMF-predictive & 0.946 & 4.703 & 1.401 & 0.903 & 3.587 & 1.002 \\
\hline$n=200$ & CVR & LEN & st.err & CVR & LEN & st.err \\
\hline nonpara-f & 0.933 & 4.222 & 1.484 & 0.888 & 3.338 & 1.151 \\
\hline nonpara-p & 0.945 & 4.594 & 1.656 & 0.900 & 3.525 & 1.249 \\
\hline trans-forward & 0.935 & 4.191 & 1.053 & 0.895 & 3.375 & 0.809 \\
\hline trans-backward & 0.935 & 4.180 & 1.047 & 0.895 & 3.375 & 0.808 \\
\hline lbs-forward & 0.930 & 4.073 & 0.967 & 0.887 & 3.272 & 0.765 \\
\hline lbs-backward & 0.930 & 4.098 & 1.080 & 0.886 & 3.292 & 0.839 \\
\hline Hybrid-trans-f & 0.926 & 4.154 & 1.485 & 0.880 & 3.322 & 1.165 \\
\hline Hybrid-trans-p & 0.938 & 4.497 & 1.650 & 0.890 & 3.475 & 1.216 \\
\hline Hybrid-lbs-f & 0.927 & 4.177 & 1.531 & 0.881 & 3.320 & 1.203 \\
\hline Hybrid-lbs-p & 0.938 & 4.496 & 1.629 & 0.893 & 3.486 & 1.228 \\
\hline MF-fitted & 0.920 & 3.830 & 0.991 & 0.871 & 3.047 & 0.728 \\
\hline MF-predictive & 0.942 & 4.369 & 1.074 & 0.893 & 3.344 & 0.817 \\
\hline SMF-fitted & 0.920 & 3.791 & 0.904 & 0.871 & 3.028 & 0.682 \\
\hline SMF-predictive & 0.945 & 4.412 & 1.076 & 0.895 & 3.350 & 0.783 \\
\hline
\end{tabular}

Table 10: Model 5: $X_{t+1}=0.75 X_{t}+0.15 X_{t} \epsilon_{t+1}+\epsilon_{t+1}$ with Laplace innovations.

Some general comments on the simulations are as follows.

- As expected, when the sample size is increased, then the coverage level accuracy is improved and the standard error associated with each interval length (denoted by st.err. in the Tables) is decreased.

- The model-based nonparametric and/or hybrid methods with predictive residuals outperform the respective ones with fitted residuals. Especially when the sample size is not large enough, using predictive residuals significantly improves the coverage level.

- The standard deviations of interval lengths are quite large for the models with heteroscedastic 
error using the model-based nonparametric and/or hybrid approaches.

- The forward and backward methods have similar performances in both the Markov bootstrap based on transition density and the local bootstrap.

- The predictive model-free (MF) methods improve the coverage level of the basic MF (denoted as MF-fitted) at the cost of higher variability.

- The smoothed model free (SMF) methods tend to have better coverage level as well as smaller standard deviations. This is analogous to the regression case discussed in eq. (6.15); see also the discussion in Section 6.6.

Comparing all the simulation results from the ten tables it is apparent that with data generated via models with homoscedastic errors, the nonparametric model-based and hybrid methods-especially the methods with predictive residuals - have better performance, particularly in view of their smaller standard deviation of the interval length; this should not be surprising since model-based methods should have an advantage when the model is true as it is the case here. Interestingly, all our Markov methods seem to be competitive with the model-based methods - even when the model is truewith the predictive SMF method being the most prominent. Finally, what is really surprising is that for data arising from models with heteroscedastic errors, all Markov bootstrap methods have better coverage level and smaller variability compared to the benchmark model-based nonparametric and/or hybrid methods.

Acknowledgement. Many thanks are due to Brendan Beare and Stathis Paparoditis for helpful discussions, and to two anonymous reviewers for constructive comments. Research of the second author was partialy supported by NSF grants DMS 13-08319 and DMS 12-23137.

\section{Appendix A: The time-reverse of a Markov process is also Markov.}

Before applying any type of backward bootstrap to a Markov process of order $p$, we need to show that the time-reverse process, i.e., the process with time-reversed sample paths, is also Markov of order $p$. For simplicity, we will assume $p=1$ throughout in this Appendix; extension to $p>1$ should be straightforward albeit notationally tedious.

Properties of Markov processes can be conveniently studied using the notion of a copula. Following Sklar (1959) and Sklar (1973), any $m$-dimensional joint distribution function $F$ with marginal distribution functions $F_{1}, F_{2}, \cdots, F_{m}$ can be written as

$$
F_{1, \cdots, m}\left(x_{1}, \cdots, x_{m}\right)=C\left(F_{1}\left(x_{1}\right), \cdots, F_{m}\left(x_{m}\right)\right)
$$

for some function $C:[0,1]^{m} \mapsto[0,1]$ called an $m$-copula. For an $m$-copula $C\left(\alpha_{1}, \cdots, \alpha_{m}\right)$, we let $C_{, j}$ denote the partial derivative $\partial C / \partial \alpha_{j}$.

Define the $\star$ operation as follows: let $A$ be an $m$-copula and $B$ be an $n$-copula. $A \star B$ is an $m+n-1$ copula given by

$$
\begin{aligned}
& A \star B\left(\alpha_{1}, \cdots, \alpha_{m+n-1}\right) \\
= & \int_{0}^{\alpha_{m}} A_{, m}\left(\alpha_{1}, \cdots, \alpha_{m-1}, \xi\right) B_{, 1}\left(\xi, \alpha_{m+1}, \cdots, \alpha_{m+n-1}\right) d \xi .
\end{aligned}
$$

Theorem A.1 (Darsow et al. (1992)). A real valued stochastic process $\left\{X_{t}, t \in T\right\}$ (where $T$ is some set of real numbers) is a Markov process if and only if for all positive integers $n$ and for all $t_{1}, \cdots, t_{n} \in T$ satisfying $t_{k}<t_{k+1}, k=1, \cdots, n-1$,

$$
C_{t_{1} \cdots t_{n}}=C_{t_{1} t_{2}} \star C_{t_{2} t_{3}} \star \cdots \star C_{t_{n-1} t_{n}},
$$


where $C_{t_{1} \cdots t_{n}}$ is the copula associated with the joint distribution of $X_{t_{1}}, \cdots, X_{t_{n}}$.

Hereafter, assume $\left\{X_{t}, t \in T\right\}$ is a Markov process. In this case, a useful property for 2-copulas and the $\star$ operation is that

$$
C_{s t}=C_{s u} \star C_{u t},
$$

for $s, u, t \in T$ satisfying $s<u<t$; cf. Theorem 3.2 of Darsow et al. (1992). We now have the following two claims that will help us show that the time-reversed process is also Markov.

Claim A.2. $C_{t_{3} t_{1}}=C_{t_{3} t_{2}} \star C_{t_{2} t_{1}}$ for any $t_{1}, t_{2}, t_{3} \in T$ and $t_{1}<t_{2}<t_{3}$.

Proof.

$$
\begin{array}{rlr}
C_{t_{3} t_{1}}\left(\alpha_{t_{3}}, \alpha_{t_{1}}\right) & =C_{t_{1} t_{3}}\left(\alpha_{t_{1}}, \alpha_{t_{3}}\right) & \text { by the definition of copula } \\
& =C_{t_{1} t_{2}}\left(\alpha_{t_{1}}, \alpha_{t_{2}}\right) \star C_{t_{2} t_{3}}\left(\alpha_{t_{2}}, \alpha_{t_{3}}\right) & \text { by eq. (A.1) } \\
& =\int_{0}^{1} C_{t_{1} t_{2}, 2}\left(\alpha_{t_{1}}, \xi\right) C_{t_{2} t_{3}, 1}\left(\xi, \alpha_{t_{3}}\right) d \xi & \text { by the definition of } \star \text { operation } \\
& =\int_{0}^{1} C_{t_{2} t_{1}, 1}\left(\xi, \alpha_{t_{1}}\right) C_{t_{3} t_{2}, 2}\left(\alpha_{t_{3}}, \xi\right) d \xi & \text { by the definition of } C_{, 1} \text { and } C_{, 2} \\
& =\int_{0}^{1} C_{t_{3} t_{2}, 2}\left(\alpha_{t_{3}}, \xi\right) C_{t_{2} t_{1}, 1}\left(\xi, \alpha_{t_{1}}\right) d \xi & \\
& =C_{t_{3} t_{2}} \star C_{t_{2} t_{1}}\left(\alpha_{t_{3}}, \alpha_{t_{1}}\right)
\end{array}
$$

Claim A.3. $C_{t_{n} \cdots t_{1}}=C_{t_{n} t_{n-1}} \star C_{t_{n-1} t_{n-2}} \star \cdots \star C_{t_{2} t_{1}}$ for all positive integers $n$ and for all $t_{1}, \cdots, t_{n} \in$ $T$ satisfying $t_{k}<t_{k+1}, k=1, \cdots, n-1$.

Proof. We prove the claim by induction.

We know that $C_{t_{3} t_{1}}=C_{t_{3} t_{2}} \star C_{t_{2} t_{1}}$ [one $\star$ operation].

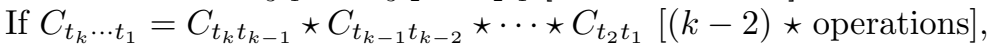

then we just need to show: $C_{t_{k+1} t_{k} \cdots t_{1}}=C_{t_{k+1} t_{k}} \star C_{t_{k} t_{k-1}} \star \cdots \star C_{t_{2} t_{1}}$.

Note that

$$
\begin{aligned}
& C_{t_{k+1} t_{k} \cdots t_{1}}\left(\alpha_{t_{k+1}}, \alpha_{t_{k}}, \cdots, \alpha_{t_{1}}\right) \\
= & C_{t_{1} \cdots t_{k} t_{k+1}}\left(\alpha_{t_{1}} \cdots, \alpha_{t_{k}}, \alpha_{t_{k+1}}\right) \\
= & \left(C_{t_{1} t_{2}} \star C_{t_{2} t_{3}} \star \cdots \star C_{t_{k-1} t_{k}}\right) \star C_{t_{k} t_{k+1}} \\
= & C_{t_{1} \cdots t_{k}} \star C_{t_{k} t_{k+1}} \\
= & \int_{0}^{\alpha_{t_{k}}} C_{t_{1} \cdots t_{k}, k}\left(\alpha_{t_{1}}, \cdots, \alpha_{t_{k-1}}, \xi\right) C_{t_{k} t_{k+1}, 1}\left(\xi, \alpha_{t_{k+1}}\right) d \xi \\
= & \int_{0}^{\alpha_{t_{k}}} C_{t_{k+1} t_{k}, 2}\left(\alpha_{t_{k+1}}, \xi\right) C_{t_{k} \cdots t_{1}, 1}\left(\xi, \alpha_{t_{k-1}}, \cdots, \alpha_{t_{1}}\right) d \xi \\
= & C_{t_{k+1} t_{k}} \star C_{t_{k} \cdots t_{1}} \\
= & C_{t_{k+1} t_{k}} \star C_{t_{k} t_{k-1}} \star \cdots \star C_{t_{2} t_{1}}
\end{aligned}
$$

by the definition of copula by Theorem A.1 by Theorem A.1 by the definition of $\star$ operation by the definition of copula by the definition of $\star$ operation by the induction assumption using $k-2$ operations 
Consider again the original Markov process $X=\left\{X_{t}, t \in T\right\}$; the time-reversed process $\tilde{X}$ is the same process but with the direction of time reversed. In particular, let $U$ be a random time point; we define the time-reversed process $\tilde{X}=\left\{\tilde{X}_{t}\right\}$ by letting $\tilde{X}_{t}=X_{U-t}$ for all $t$ such that $U-t \in T$. We are now able to show that the time-reversed process is also Markov.

Lemma A.4. The time-reverse of a Markov process is also a Markov process.

Proof. The proof is immediate by Claims A.2 and A.3 in connection with Theorem A.1.

\section{Appendix B: Prediction intervals in $r$-step-ahead prediction.}

As before, let $X_{1}=x_{1}, X_{2}=x_{2}, \cdots, X_{n}=x_{n}$ denote the observed sample path from the $p$-th order Markov chain $X$, and let $y_{n}=\left(x_{n}, \cdots, x_{n-p+1}\right)^{\prime}$. The objective now is point and interval prediction of $X_{n+r}$ given the data where $r$ is a positive integer.

The case $r=1$ was extensively discussed in the main paper, and several different methods were developed, including forward and backward bootstrap methods. Interestingly, when $r>1$, all forward and backward bootstrap methods are readily applicable with minimal modifications. The only exception is the hybrid method, the reason being that $r$-step-ahead prediction is cumbersome using a one-step-ahead model equation such as (5.1) or (5.2).

To start, note that the $L_{2}$-optimal predictor of $X_{n+r}$ is given by the conditional expectation $E\left(X_{n+r} \mid X_{n}, \ldots, X_{1}\right)=E\left(X_{n+r} \mid X_{n}, \ldots, X_{n-p+1}\right)$; the latter simplification is due to the Markov property. As before, we can approximate the theoretical $L_{2}$-optimal predictor via several differentbut asymptotically equivalent - methods, e.g.

$$
\hat{X}_{n+r}=\int x \hat{f}_{n, r}\left(x \mid y_{n}\right) d x \text { or } \hat{X}_{n+r}=\hat{m}_{r}\left(y_{n}\right) ;
$$

here, $\hat{f}_{n, r}\left(x \mid y_{n}\right)$ is a kernel smoothed estimate of the conditional density of $X_{n+r}$ given $\left(X_{n}, \ldots, X_{n-p+1}\right)^{\prime}=y_{n}$, and $\hat{m}_{r}(y)$ is a kernel estimator of the regression on the scatterplot of $X_{n+r}$ vs. $\left(X_{n}, \ldots, X_{n-p+1}\right)^{\prime}$, i.e.,

$$
\hat{m}_{r}(y)=\frac{\sum_{t=p}^{n-r} K\left(\frac{\left\|y-y_{t}\right\|}{h}\right) x_{t+r}}{\sum_{t=p}^{n-r} K\left(\frac{\left\|y-y_{t}\right\|}{h}\right)}
$$

with the understanding that $p+r$ is (much) smaller than $n$ in order for $\hat{m}_{r}(y)$ and $\hat{f}_{n, r}\left(x \mid y_{n}\right)$ to be accurate estimators.

As regards prediction intervals, the three methods go as follows.

Algorithm B.1. Forward Bootstrap based on transition density for $r$-step-ahead prediction

(1) Choose a probability density $K$ on $\mathbb{R}^{2}$ and positive bandwidths $h_{1}, h_{2}$ to compute $\hat{f}_{n}(y)$ and $\hat{f}_{n}(x \mid y)$ from eq. (3.2) and (3.3) respectively. Also construct the following kernel estimators:

$$
\begin{aligned}
\hat{f}_{n, r}(x, y) & =\frac{1}{(n-p-r+1) h_{1} h_{2}} \sum_{i=p}^{n-r} K\left(\frac{x-x_{i+r}}{h_{1}}, \frac{\left\|y-y_{i}\right\|}{h_{2}}\right) \\
\hat{f}_{n, r}(x \mid y) & =\frac{\hat{f}_{n, r}(x, y)}{\hat{f}_{n, r}(y)} .
\end{aligned}
$$


(2) Calculate the point predictor $\hat{x}_{n+r}=\int x \hat{f}_{n, r}\left(x \mid y_{n}\right) d x$; alternatively, use the equivalent estimate of conditional expectation from eq. (B.1).

(3) Steps (a)-(c) are as in Algorithm 3.1.

(d) Compute $\hat{f}_{n}^{*}(x \mid y)$ and $\hat{f}_{n, r}^{*}(x \mid y)$ in a similar way as in eq. (3.3) and (B.4) respectivelywith the same kernel and bandwidths - but based on the pseudo-data $x_{1}^{*}, x_{2}^{*}, \cdots, x_{n}^{*}$ instead of the original data.

(e) Calculate the bootstrap point predictor $\hat{x}_{n+r}^{*}=\int x \hat{f}_{n, r}^{*}\left(x \mid y_{n}\right) d x$.

(f) Generate the bootstrap future value $x_{n+r}^{*} \sim \hat{f}_{n, r}\left(\cdot \mid y_{n}\right)$.

(g) Calculate the bootstrap root replicate as $x_{n+r}^{*}-\hat{x}_{n+r}^{*}$.

(4) Repeat (3) B times; the B bootstrap root replicates are collected in the form of an empirical distribution whose $\alpha$-quantile is denoted $q(\alpha)$.

(5) The $(1-\alpha) 100 \%$ equal-tailed, bootstrap prediction interval for $X_{n+r}$ is given by

$$
\left[\hat{x}_{n+r}+q(\alpha / 2), \hat{x}_{n+r}+q(1-\alpha / 2)\right] \text {. }
$$

As apparent from the above, the one-step-ahead transition density $\hat{f}_{n}(x \mid y)$ is used to generate the pseudo-data $x_{1}^{*}, x_{2}^{*}, \cdots, x_{n}^{*}$ while the $r$-step-ahead transition density $\hat{f}_{n, r}(x \mid y)$ is reserved for extrapolation purposes, i.e., to yield the bootstrap point predictor and the bootstrap future value.

Algorithm B.2. Backward Bootstrap based on transition density for $r$-step-ahead prediction The algorithm is identical to Algorithm B.1 with the following exception: steps 3 (a)-(c) should be taken as the corresponding steps in Algorithm 3.2.

A similar construction can be employed in the Local Bootstrap. As before, we can compute the onestep-ahead empirical distribution estimator $\tilde{F}_{n}(x \mid y)$ from eq. (4.1), and the $r$-step-ahead empirical distribution estimator

$$
\tilde{F}_{n, r}(x \mid y)=\frac{\sum_{j=p}^{n-r} 1_{(-\infty, x]}\left(x_{j+r}\right) W_{b}\left(y-y_{j}\right)}{\sum_{m=p}^{n-r} W_{b}\left(y-y_{m}\right)},
$$

but the Local Bootstrap algorithm is easier to implement using the probability mass functions associated with these two discrete distributions.

Algorithm B.3. Forward Local Bootstrap for r-step-ahead prediction

(1) Choose a resampling kernel $W$ and bandwidth $b$, and compute the the predictor $\hat{x}_{n+r}$ as

$$
\frac{\sum_{j=p}^{n-r} W_{b}\left(y_{n}-y_{j}\right) x_{j+r}}{\sum_{m=p}^{n-r} W_{b}\left(y_{n}-y_{m}\right)} .
$$

(2) Steps (a)-(c) are as in Algorithm 4.1.

(d) Calculate the bootstrap predictor $\hat{x}_{n+r}^{*}$ as

$$
\frac{\sum_{j=p}^{n-r} W_{b}\left(y_{n}-y_{j}^{*}\right) x_{j+r}^{*}}{\sum_{m=p}^{n-r} W_{b}\left(y_{n}-y_{m}^{*}\right)},
$$

where $y_{t}^{*}=\left(x_{t}^{*}, \cdots, x_{t-p+1}^{*}\right)^{\prime}$. 
(e) Re-define $y_{n}^{*}=y_{n}$, and then generate $x_{n+r}^{*}=x_{J+r}$ as in step (b), where $J$ is a discrete random variable taking its values in the set $\{p, \cdots, n-1\}$ with probability mass function given by

$$
P(J=s)=\frac{W_{b}\left(y_{n}-y_{s}\right)}{\sum_{m=p}^{n-r} W_{b}\left(y_{n}-y_{m}\right)} .
$$

(f) Calculate the bootstrap prediction root replicate as $x_{n+r}^{*}-\hat{x}_{n+r}^{*}$.

(3) Repeat step (2) B times; the B bootstrap root replicates are collected in the form of an empirical distribution whose $\alpha$-quantile is denoted $q(\alpha)$.

(4) The $(1-\alpha) 100 \%$ equal-tailed, forward Local Bootstrap prediction interval for $X_{n+r}$ is given by

$$
\left[\hat{x}_{n+r}+q(\alpha / 2), \hat{x}_{n+r}+q(1-\alpha / 2)\right] .
$$

Algorithm B.4. Backward Local Bootstrap for r-step-ahead prediction

The algorithm is identical to Algorithm B.3 with the following exception: steps 3 (a)-(c) should be taken as the corresponding steps in Algorithm 4.2.

Finally, we discuss the Model-Free Bootstrap methods as they apply to $r$-step-ahead prediction. To do this, we need to define the extrapolation estimated distributions as follows. Define

$$
\hat{C}_{y}(x)=\frac{\sum_{i=p}^{n-r} 1_{\left\{x_{i+r} \leq x\right\}} K\left(\frac{\left\|y-y_{i}\right\|}{h}\right)}{\sum_{k=p}^{n-r} K\left(\frac{\left\|y-y_{k}\right\|}{h}\right)},
$$

and let $\tilde{C}_{y}(x)$ be a continuous version of $\hat{C}_{y}(x)$ obtained by linear interpolation. Also let

$$
\bar{C}_{y}(x)=\frac{\sum_{i=p}^{n-r} \Lambda\left(\frac{x-x_{i+r}}{h_{0}}\right) K\left(\frac{\left\|y-y_{i}\right\|}{h}\right)}{\sum_{k=p}^{n-r} K\left(\frac{\left\|y-y_{k}\right\|}{h}\right)} .
$$

Note that $\hat{C}_{y}(x), \tilde{C}_{y}(x)$ and $\bar{C}_{y}(x)$ are all estimates of $C_{y}(x)=P\left(X_{t+r} \leq x \mid Y_{t}=y\right)$.

Algorithm B.5. Model-Free Bootstrap for r-step-ahead prediction

The basic MF, SMF, PMF and SPMF algorithms are identical to Algorithms 6.3, 6.2, 6.3 and 6.4 respectively with the following changes that pertain only to the calculation of the real-world predictor $\hat{x}_{n+r}$, bootstrap predictor $\hat{x}_{n+r}^{*}$ and bootstrap future value $x_{n+r}^{*}$.

Cases MF and PMF: replace $\hat{D}_{y_{n}}(\cdot)$ and $\tilde{D}_{y_{n}}(\cdot)$ by $\hat{C}_{y_{n}}(\cdot)$ and $\tilde{C}_{y_{n}}(\cdot)$ respectively.

Cases SMF and SPMF: replace $\bar{D}_{y_{n}}(\cdot)$ by $\bar{C}_{y_{n}}(\cdot)$.

\section{References}

Alonso, A. M., Peña, D., and Romo, J. (2002). Forecasting time series with sieve bootstrap. Journal of Statistical Planning and Inference, 100(1):1-11.

Box, G. E. and Jenkins, G. M. (1976). Time series analysis, control, and forecasting. San Francisco, CA: Holden Day.

Breidt, F. J., Davis, R. A., and Dunsmuir, W. (1995). Improved bootstrap prediction intervals for autoregressions. Journal of Time Series Analysis, 16(2):177-200. 
Carroll, R. J. and Ruppert, D. (1991). Prediction and tolerance intervals with transformation and/or weighting. Technometrics, 33(2):197-210.

Darsow, W. F., Nguyen, B., and Olsen, E. T. (1992). Copulas and markov processes. Illinois Journal of Mathematics, 36(4):600-642.

Geisser, S. (1993). Predictive inference: an Introduction. Chapman and Hall: New York.

Kreiss, J.-P. and Neumann, M. H. (1998). Regression-type inference in nonparametric autoregression. The Annals of Statistics, 26(4):1570-1613.

Li, Q. and Racine, J. S. (2007). Nonparametric econometrics: Theory and practice. Princeton University Press.

Masarotto, G. (1990). Bootstrap prediction intervals for autoregressions. International Journal of Forecasting, 6(2):229-239.

Olive, D. J. (2007). Prediction intervals for regression models. Computational statistics and data analysis, 51(6):3115-3122.

Olive, D. J. (2015). Highest Density Region Prediction. manuscript available from: http://lagrange.math.siu.edu/Olive/pphdrpred.pdf.

Pan, L. (2013). Bootstrap prediction intervals for time series. Ph.D. Thesis, Department of Mathematics, University of California-San Diego.

Pan, L. and Politis, D. N. (2015). Bootstrap prediction intervals for linear, nonlinear and nonparametric autoregressions (with discussion). Journal of Statistical Planning and Inference, to appear.

Paparoditis, E. and Politis, D. N. (1998). The backward local bootstrap for conditional predictive inference in nonlinear time series. 4th Hellenic-European Conference on Computer Mathematics and its Applications (HERCMA'98), E. A. Lipitakis (Ed.), Lea Publishing, Athens, Greece, pages 467-470.

Paparoditis, E. and Politis, D. N. (2002). The local bootstrap for markov processes. Journal of Statistical Planning and Inference, 108(1):301-328.

Pascual, L., Romo, J., and Ruiz, E. (2004). Bootstrap predictive inference for arima processes. Journal of Time Series Analysis, 25(4):449-465.

Patel, J. (1989). Prediction intervals-a review. Communications in Statistics-Theory and Methods, 18(7):2393-2465.

Politis, D. N. (2010). Model-free model-fitting and predictive distributions. UCSD Dept. of Economics Discussion Paper, URL:http://www.escholarship.org/uc/item/67j6s174.

Politis, D. N. (2013). Model-free model-fitting and predictive distributions. Test, 22(2):183-221.

Rajarshi, M. (1990). Bootstrap in markov-sequences based on estimates of transition density. Annals of the Institute of Statistical Mathematics, 42:253-268.

Rosenblatt, M. (1952). Remarks on a multivariate transformation. The Annals of Mathematical Statistics, pages $470-472$.

Schmoyer, R. L. (1992). Asymptotically valid prediction intervals for linear models. Technometrics, 34(4):399-408.

Sklar, A. (1959). Fonctions de répartition à $n$ dimensions et leurs marges. vol. 8, Publ. Inst. Statist. Université Paris.

Sklar, A. (1973). Random variables, joint distribution functions, and copulas. Kybernetika, 9(6):449460.

Stine, R. A. (1985). Bootstrap prediction intervals for regression. Journal of the American Statistical Association, 80(392):1026-1031.

Thombs, L. A. and Schucany, W. R. (1990). Bootstrap prediction intervals for autoregression. Journal of the American Statistical Association, 85(410):486-492.

Wolf, M. and Wunderli, D. (2015). Bootstrap joint prediction regions. Journal of Time Series 
Analysis, 36(3):352-376. 